\title{
OPTEER, un dispositif de connaissance et d'analyse territoriale par et pour les acteurs de la transition énergétique.
}

\author{
Marie-H. de Sède-Marceau' ${ }^{1}$, Stéphane François ${ }^{2}$, Benjamin Pauc ${ }^{2}$, \\ 1. Laboratoire ThéMA, UMR-6049, CNRS et Université de Bourgogne Franche- \\ Comté \\ 32, rue Mégevand, F-25030 Besançon, France \\ Marie-helene.de-sede-marceau@univ-fcomte.fr \\ 2. ATMO Franche-Comté \\ 15 rue Mégevand \\ 25000 Besançon, France
}

RESUME. Cet article a pour objectif d'illustrer, à travers la mise en æuvre et l'exploitation de la plateforme OPTEER (Observation Prospective Territoriale Energétique à l'Echelle Régionale), comment sont utilisés les différents outils mis à disposition des acteurs territoriaux et quels usages collectifs de l'information géographique se sont développés. Sur la base de cette expérience de plus de 10 ans, autour de laquelle se sont cristallisés développements opérationnels et expérimentations de recherche, sont également évoqués les nouveaux besoins liés aux exigences de la mise en ouvre de la transition énergétique et les pistes de recherche, de développement et de portage envisagées pour garantir la pérennité du dispositif.

ABSTRACT. The aim of this article is to illustrate, through the implementation and exploitation of the OPTEER platform (Territorial energy observation and Prospective at the regional scale), how the various tools made available to territorial actors and what collective uses of geographic information have developed. Based on his experience of more than 10 years, around which crystallized operational developments and research experiments, are also evoked the new needs related to the requirements of the implementation of energy transition and the tracks of research, development and porting envisaged to guarantee the durability of the device.

Mots-clés: Usages de l'information, observation territoriale, prospective territoriale, données climat-air-énergie, animation, systèmes d'information.

KEYWORDS: Uses of information, territorial observation, territorial foresight, climate-airenergy data, animation, information systems. 


\section{Introduction}

Les mécanismes conduisant d'une part au changement climatique et d'autre part à la dégradation de la qualité de l'air sont conditionnés par nos consommations énergétiques tant d'un point de vue quantitatif que qualitatif. Ces consommations sont elles-mêmes fortement dépendantes des modes d'organisation et de fonctionnement de nos sociétés dans leurs territoires ${ }^{1}$. Maitriser l'espace en améliorant par exemple l'accessibilité, est énergivore, tout comme garantir et renforcer les équilibres socio-économiques garant d'un développement que nous souhaitons durable. Nos mobilités, nos façons d'habiter, de produire, de consommer ${ }^{2}$, l'ensemble de nos activités représentées par ces secteurs concourent au bilan énergétique et environnemental de nos territoires.

C'est pourquoi, au-delà d'une nécessaire comptabilisation des productions et des consommations énergétiques, les diagnostics, scénarios et orientations doivent intégrer la diversité structurelle et fonctionnelle des territoires et les usages de l'énergie qui en découlent. Cette territorialisation ${ }^{3}$ permet l'articulation entre orientations globales, régionales et locales, et ce, jusqu'à la mise en œuvre de projets opérationnels. Elle facilite l'identification des variables endogènes et des leviers d'actions permettant d'influer sur le système énergétique. Elle ouvre la voie à l'identification d'objectifs ciblés et à la mise en œuvre d'actions territoriales différenciées selon les zones, mais complémentaires au regard des ambitions territoriales.

Qu'implique cette démarche ? Tout d'abord la prise de conscience de la complexité des enjeux et des défis qui ne peuvent être relevés sans la mobilisation de l'ensemble des acteurs du territoire. Ensuite, elle appelle à une meilleure compréhension de la complexité du système socio-spatial, de son degré de dépendance avec l'extérieur, nécessitant, au-delà de la production de diagnostics sectoriels, le développement d'approches transversales, dépassant les visions «métiers ». Elle impose la mise en œuvre d'une véritable stratégie informationnelle collective et de long terme, capable d'alimenter les diagnostics attendus et de renseigner sur les évolutions en cours par le biais d'outils de suivi, d'analyse et de prospective appuyés par un ensemble de dispositifs d'animation et d'accompagnement. Elle contribue enfin à l'émergence de

\footnotetext{
${ }^{1}$ Organisation dont la maîtrise, partielle, peut passer par l'évolution de la législation. On peut citer par exemple le volet urbanisme de la loi ALUR qui pour faciliter notamment la densification des quartiers pavillonnaires, supprime la disposition de la loi Urbanisme et Habitat de 2003 qui permettait au plan local d'urbanisme (PLU) de fixer une taille minimale de terrain, ainsi que le coefficient d'occupation des sols (COS).

${ }^{2} \mathrm{Y}$ compris en intégrant l'énergie grise correspondant à la dépense énergétique totale pour l'élaboration d'un matériau, de son extraction à son recyclage, qui pose des problèmes de prise en compte à une échelle territoriale flexible.

${ }^{3}$ La territorialisation implique de fournir au travers ou en complément de la donnée énergétique des éléments de contexte et de compréhension de la nature des facteurs qui interviennent dans la réalité observée du territoire (Mazaud et al, 2017).
} 
nouvelles représentations des territoires comme des enjeux qui leurs sont attachés et opèrent ainsi une véritable mutation cognitive des acteurs territoriaux (Lardon et al. 2006).

La plateforme OPTEER (Observation Prospective Territoriales Energétique à l'Echelle Régionale) s'inscrit dans cette philosophie. Conçue et prototypée par une équipe de recherche en géographie, en collaboration et avec le soutien financier de l'ADEME (Agence De l'Environnement et de la Maîtrise de l'Energie) et de la Région Franche-Comté, ainsi que de l'agglomération du Grand Dole, puis portée par ATMO Franche-Comté (ATMO-FC) ${ }^{4}$, OPTEER a pour ambition de développer des outils d'observation et des méthodes d'analyse et de prospective afin d'accompagner les décideurs et plus largement l'ensemble des acteurs du territoire dans leurs démarches d'analyse et de planification territoriales dans le cadre de la transition énergétique.

Opérationnel depuis 2010, OPTEER (http://www.opteer.org/) met à disposition de ses partenaires un observatoire énergétique territorial dont les fonctionnalités permettent l'observation et l'analyse du territoire abordé sous l'angle air-énergieclimat. L'ensemble des missions et des fonctionnalités qui lui sont attachées nécessitent ainsi un suivi en termes de données, la mobilisation d'un ensemble élargi de partenaires, et une veille méthodologique et technologique constants.

Ainsi, OPTEER ne peut être uniquement rapporté à un outil technologique. Les réflexions menées sur les trois années de conception, puis à partir de sa mise en production en 2010 et l'augmentation constante des utilisateurs et des usages faits des informations qu'il produit montrent l'intérêt de l'ensemble des éléments mis en œuvre, couplant système d'information et dispositif d'animation et d'accompagnement.

Après une présentation de la plateforme OPTEER et des contextes informationnel, institutionnel, sociétal et environnemental dans lesquels s'est inscrit son développement, nous verrons comment les acteurs territoriaux se sont appropriés l'ensemble du dispositif et le font vivre et quels usages des informations produites se sont développés, traduisant notamment les besoins des petites collectivités en matière de réalisation et de suivi des différents documents d'urbanismes, plans et schémas visant à favoriser l'atteinte des objectifs en matière d'énergie et de climat (Godinot, 2014). Enfin, sur la base d'une prise de recul critique de cette expérience, nous évoquerons les perspectives d'évolution envisagées aujourd'hui après 7 années d'exploitation.

\footnotetext{
${ }^{4}$ ATMO Franche-Comté : Association de l'environnement atmosphérique (Air-EnergieClimat) en Franche-Comté (voir : http://www.atmo-franche-comte.org/)
} 


\section{Cadre général et philosophie de l'approche de conception, de développement et de portage du dispositif OPTEER}

\subsection{Le contexte et la problématique}

La transition énergétique se traduit dans les faits, pour les collectivités comme pour les individus par une avalanche de lois, injonctions, par une complexification des pratiques de gestion et d'aménagement des territoires (Souami, 2007 ; Poupeau, 2013) et de l'environnement et plus globalement par une croissance continue d'informations variées scientifiques, techniques, opérationnelles, législatives, issues d'études, d'audit, etc.

Dans ce contexte, il n'est donc pas étonnant, au carrefour des problématiques territoriales et énergétiques, de voir émerger de très fortes attentes en matière de diagnostics et de prospective énergétiques du côté des collectivités et des institutions en charge de piloter la transition attendue. Ces attentes sont fortement conditionnées par le contexte législatif avec, notamment, la promulgation de la loi sur la Transition Energétique $^{5}$, la stratégie nationale bas carbone, les dispositifs régionaux tels que les SRCAE (Schémas Régionaux Climat Air Énergie), locaux avec les PCAET (Plans Climat Air Énergie Territoriaux), ou encore avec les SDE (Schéma directeurs de l'énergie). Ces attentes se lisent également à travers les nombreux programmes de recherche/innovation (programmes nationaux type ANR (Agence Nationale de la Recherche), Reference Framework for European Sustainable cities, Villes et communautés intelligentes) et initiatives d'acteurs publics et privés (Convention des maires à l'échelle des villes européennes par exemple).

Ces évolutions révèlent l'importance des enjeux liés à la maîtrise de l'information territoriale et placent de fait les dispositifs et les outils techniques de collecte, gestion et analyse de données au cœur du cycle territoires-informationaction/décision.

En matière d'aménagement et de développement territorial, le rôle des TIC (Technologies de l'Information et de la Communication) et plus spécifiquement des TIG (Technologies de l'information Géographique ${ }^{6}$ ) est depuis longtemps reconnu (Pornon, 1998 ; Roche et al. 1996). Leur usage abouti aujourd'hui à la multiplication de systèmes d'information de type Infrastructures de Données Géographique (IDG), observatoires, outils de scénarisation, notamment à l'initiative de l'État ou des collectivités et faisant l'objet d'études diverses (Noucher et al., 2016). D'un point de vue énergétique, l'émergence d'approches décentralisées et la promotion de mix

\footnotetext{
${ }^{5}$ LOI n ${ }^{\circ}$ 2015-992 du 17 août 2015 relative à la transition énergétique pour la croissance verte

${ }^{6}$ Les technologies de l'information géographique regroupent l'ensemble des outils, techniques et méthodes permettant de représenter, de manipuler et d'analyser des données géographiques et de produire et véhiculer des représentations spatiales (cartographie assistée par ordinateur, système d'information géographique, services Web cartographiques, télédétection, GPS) (Méricskay, 2011).
} 
énergétiques favorisent la diffusion de nouveaux dispositifs essentiellement techniques de gestion et de planification énergétique de type smart-grids ${ }^{7}$ reposant sur l'utilisation des TIC. Ils offrent des possibilités élargies en termes d'amélioration du confort et de l'efficacité économique et environnementale des villes (Chérix et al. 2013). De nombreux acteurs en font d'ailleurs un axe de développement stratégique comme l'illustre le Scénario GRDF (Gaz Réseau Distribution France) Facteur $4^{8}$. Enfin, les potentiels de collaboration et de coproduction offerts par Internet contribuent au développement de systèmes d'information collaboratifs (Mericskay, 2011; Sui et al., 2012) à l'instar de la plateforme pour la géothermie ${ }^{9}$ ou d'OpenStreetMap, posant légitimement la question de la qualité des informations produites (Haklay, 2010 ; Palsky G. 2013 ; Joliveau et al. 2013).

Certains dispositifs sont opérationnels depuis de nombreuses années permettant l'analyse de leurs usages et impacts tant sur les systèmes territoriaux qu'au niveau des politiques mises en œuvre et des objectifs visés. Ces réflexions amènent à s'interroger sur les modes de fonctionnement organisationnels en place autour des outils, la construction des légitimités d'action, les rôles de l'information et de la connaissance des territoires sur celles-ci, faisant de ces dispositifs sociotechniques de véritables objets de recherche (Noucher, 2013).

OPTEER accompagne les acteurs territoriaux francs-comtois ${ }^{10}$ depuis 7 ans, alimentant leurs réflexions et leurs actions dans les domaines de l'énergie, de la qualité de l'air et du climat. Les dynamiques institutionnelles en cours, notamment dans le cadre de la fusion des Régions nous ouvrent aujourd'hui une fenêtre d'opportunité favorisant une prise de recul et une analyse circonstanciée des évolutions du dispositif et de ses usages mais aussi une réflexion prospective sur son rôle dans la dynamique de transition engagée.

\subsection{Fondements théoriques et conceptuels à la base des spécifications et du développement de l'outil OPTEER}

Pour parvenir à approcher l'énergie indispensable, omniprésente et organisatrice du territoire, une lecture systémique et territorialisée des liens territoire-énergie a été mise en œuvre lors de la conception de l'outil OPTEER. L'ambition était de traduire au mieux les interactions entre un territoire et son système énergétique, et plus

\footnotetext{
${ }_{8}^{7}$ Smart grid : réseau électrique intelligent

http://bibliotheque.grdf.fr/fileadmin/user_upload/images/actualites/DNTE_scenarioGrDF_Fac teur_4_avril2013.pdf

https://www.wien.gv.at/umweltgut/public/grafik.aspx?bookmark=NfGWReYdgkWC7tNGTU NDRjnC-cs6-crOsX3Z-cJ1e1MUTLgxnQ4HcuJ-ax7VLUGutcR6jPaPzYYMoOOJCahxLmIC1A8-b

${ }^{10}$ Collectivités locales, associations, bureaux d'études, services de l'Etat
} 
précisément, la dépendance réciproque des processus énergétiques et des processus territoriaux afin d'assurer une planification et une gestion déclinées et adaptées à un contexte local, appropriables par les acteurs du territoire. Par ailleurs et au-delà d'une entrée purement théorique, il a été nécessaire de comprendre les fonctionnements des systèmes énergétiques et territoriaux à travers l'étude des outils utilisés pour leurs planifications. Du point de vue des territoires, cette étude des outils de planification territoriale a conclu à l'intégration progressive des problématiques énergétiques dans les documents opérationnels (Desjardin, 2007 ; CERTU, 2011). L'analyse de modèles énergétiques classiquement utilisés en planification énergétique a par ailleurs validé l'idée pressentie d'une prédominance des approches technico-économiques ou sectorielles (Mazaud et al., 2017), considérant des «flux hors-sols » (Jebaraj, 2006). Cependant les ambitions affichées dans le cadre du prototypage d'OPTEER imposaient de pouvoir passer d'états des lieux effectués par une comptabilité énergétique, à un niveau de compréhension des processus en jeu et d'en identifier des leviers d'action. Cette perspective passait par une meilleure prise en compte du contexte et des spécificités territoriales impactant le comportement énergétique du système territoire.

Cette approche a représenté un véritable défi en termes de connaissance. En effet, malgré la multiplication et l'apparente abondance de données et informations disponibles, nous n'avons pu que constater les difficultés à parvenir à une vision intégrée des transformations structurelles et fonctionnelles des territoires et de leurs liens à l'énergie, à différentes échelles. Ainsi, contrairement à de nombreux autres projets d'observatoires (Chabret, 2014), le développement de l'outil «technique » OPTEER $^{11}$ a précédé le dispositif qui s'est, quant à lui, construit au fil du temps avec la mise en relation puis en synergie des partenaires (fournisseurs de données, collectivités, agences, ...) via un processus d'animation territoriale (voir historique développé dans le 3.1).

Ces différents éléments nous ont donc amené à travailler dans un premier temps sur la dimension informationnelle du projet et à adopter une démarche de conception centrée sur la définition de concepts à l'interface entre représentation du système énergétique territorial et données énergétiques et géographiques (cf. figure 1). Nous avons ainsi proposé un certain nombre de solutions sémantiques, structurelles et fonctionnelles favorisant l'intégration et la mise en cohérence de données hétérogènes pour la production d'indicateurs énergétiques territoriaux (Fléty et al., 2009 ; Fléty, 2014).

Comme le montre la figure 1, l'approche a reposé sur le recours aux principes des ontologies de domaine pour construire une ontologie du domaine «territoireénergie». Les acceptions du terme ontologie sont multiples (Guarino, 1998; Spaccapietra, 2004 ; Flety, 2009 ; Couclelis, 2010). Dans le cadre de ce projet, nous avons défini cette géo-ontologie à la fois comme une représentation du système

\footnotetext{
${ }^{11}$ Par outil technique nous entendons la conception (choix sémantiques, de formalisation et de modélisation) et le développement (langages, outils, architecture techniques) du système d'information
} 
observé et comme un système de référence sémantique pour l'information territoriale énergétique, celle-ci étant donc considérée comme un pont sémantique entre le réel et la donnée (cf. figure 2).

Ce choix permet d'améliorer les capacités d'explicitation, d'intégration et de transposition de concepts. En outre, l'ontologie proposée constitue un objet de médiation dans le processus de construction de connaissance, particulièrement pertinent dans un contexte collaboratif de construction d'observatoire territorial multi-acteurs (Fléty, 2014).

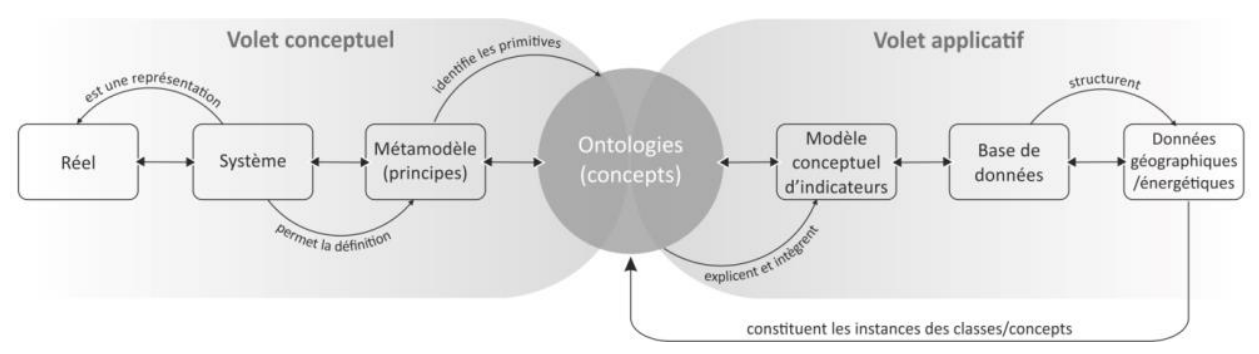

Figure 1 : Démarche de conception du prototype OPTEER (source : Fléty, 2014)

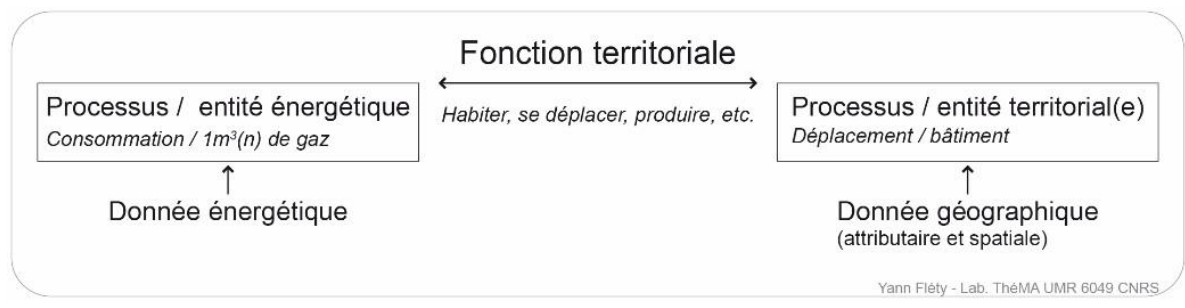

Figure 2 : Une entrée par les fonctions territoriales et une lecture énergétique d'entités territoriales (Source : Fléty, 2014)

Une fois les choix opérés tant d'un point de vue sémantique et conceptuel qu'en termes d'architecture de données, il était nécessaire de régler différentes questions d'ordre technique, liées notamment à la volumétrie des données à maîtriser, leur croissance attendue ainsi qu'aux exigences de qualité et de normes qu'imposent la nécessaire interopérabilité de ce type de système d'information. Ainsi, dans un souci de compatibilité avec les grands standards du marché de développement d'application informatique et de conformité à la directive européenne INSPIRE, l'application a été développée sur la base d'une technologie compatible en termes d'hébergement et d'échange de données. 


\subsection{Questions autour des besoins et usages pressentis de la plateforme OPTEER}

Les options techniques et les principes théoriques et conceptuels posés et validés dans le cadre des spécifications puis des développements du prototype, le travail de réflexion organisationnelle a pris le dessus, à partir de 2010, date de la mise à disposition de la plateforme aux utilisateurs. Ces réflexions ont été guidées par un certain nombre d'éléments et parfois de ressentis quant à l'évolution des besoins de connaissance des territoires par les acteurs de leurs évolutions, qu'ils dépendent des sphères publiques, associatives ou privées. Plusieurs points ont attiré notre attention :

2.3.1. La question de l'ingénierie territoriale et de l'accompagnement/des services personnalisés aux collectivités

De nombreux travaux interrogent aujourd'hui les moyens dont disposent les acteurs territoriaux pour comprendre, analyser et décider. L'ingénierie territoriale, visant à produire, mobiliser, mutualiser les informations pour améliorer la connaissance des territoires (Janin et al. 2011 ; Trognon et al. 2012) répond-elle aux besoins croissants exprimés par les acteurs dans ce domaine ? Sous quelles conditions? Ces questions sont d'autant plus cruciales que l'évolution des cadres législatifs offrent de nouvelles perspectives en matière de compréhension des systèmes énergétiques territoriaux ${ }^{12}$ et amènent aujourd'hui les collectivités à se réapproprier la maitrise de certains choix en matière énergétique notamment. On peut penser ici aux démarches TEPOS (Territoires à Energie POSitive), TEPCV (Territoires à Energie Positive pour la Croissance Verte). Dans ce contexte, il était important de définir la place du dispositif OPTEER et notamment d'identifier ce que les acteurs francs comtois en attendaient, quels usages de la plateforme OPTEER pouvait on anticiper? Les nombreuses réflexions menées à la faveur du lancement de la plateforme nous ont rapidement convaincu de la nécessité d'instaurer un accompagnement des utilisateurs à géométrie variable. Il s'est avéré nécessaire de répondre aux attentes de chacun, en proposant un accompagnement et des services adaptés aux petites collectivités non dotées de compétences d'ingénierie territoriale, en parallèle de la fourniture de données et de fonctionnalités de traitement, d'analyse et de représentation.

\subsubsection{La question de la qualité et de la sécurisation des données}

Au-delà de la nécessité d'un contrôle de la qualité des données intégrées à l'outil OPTEER, le rôle stratégique des métadonnées « capables d'abstraire et de capturer les informations essentielles sur les données sous-jacentes, indépendamment de détails représentatifs », (Kashyap, 1995) est rapidement apparu. L'intégration de

\footnotetext{
${ }^{12}$ Avec notamment les évolutions réglementaires dans le cadre de la loi TECV (article 179 sur les données énergétiques), concernant les données de transport et de distribution de gaz naturel, d'électricité, tant pour la consommation que pour la production (bio-méthane, électricité renouvelable) ainsi que les réseaux de chaleur.
} 
données hétérogènes multi-sources, la coproduction d'indicateurs composites et leur diffusion au-delà des sphères métiers à leur origine, imposaient la définition d'un cadre rigoureux permettant rapidement aux utilisateurs de disposer des éléments leur permettant de juger de la qualité, de la fiabilité, de la pertinence des données. La plateforme OPTEER, se devait donc d'offrir la possibilité de renseigner des métadonnées sur des contenus de tous types, intégrés dans l'outil ou produits dans l'environnement de l'observatoire (formules, cartes, graphiques, ...). Au-delà des informations de base (producteur, unité, description de la donnée, ...), il apparaissait nécessaire de préciser les limites ou encore les précautions d'utilisation de la donnée, de l'indicateur, de la carte ou du graphique et d'attacher automatiquement les métadonnées aux fichiers de données lors des exports, le tout dans le respect de la directive INSPIRE.

Mais d'autres questions inhérentes au caractère stratégique des données détenues par certains partenaires et aux cadres réglementaires en vigueur se sont rapidement posées (législation sur les Informations Commercialement Sensibles (ICS) par exemple). Les partenariats initiés très tôt avec certains opérateurs énergétiques ainsi qu'avec des acteurs industriels d'envergure nationale, voire au-delà, ont ainsi permis de mettre en œuvre un certain nombre de réflexions autour de la sécurisation des données et favorisé le développement de solutions aujourd'hui opérationnelles. Ces efforts restent un véritable « atout confiance » auprès des partenaires.

\subsubsection{La question de la dynamique des acteurs et de l'intensité de leur collaboration}

La capacité à développer des outils communs et à produire de l'information sous la forme d'indicateurs élaborés, construits à partir de données provenant de divers domaines/partenaires nous semble être, au-delà des dimensions technologiques et méthodologiques un véritable catalyseur favorable au renforcement et à l'enrichissement des modes de gouvernance existants dans les territoires. Dans ce cadre, les indicateurs composites produits par les dispositifs tel qu'OPTEER deviennent alors des révélateurs du niveau de collaboration entre les partenaires (de Sède-Marceau et al., 2011). Il a donc été important, lors de la phase de prototypage de développer un outil évolutif permettant de garantir cette capacité de coproduction mais aussi de prise en compte de l'évolution des besoins et des acteurs confrontés aux problématiques énergétiques et à la mise en œuvre des transitions attendues. Seules ces exigences permettent d'en assurer la pérennité dans le temps, au fil des adhésions nouvelles au dispositif, de l'apport de nouvelles données mais aussi des évolutions législatives et sociétales (notamment des systèmes sociotechniques).

\section{Le dispositif OPTEER}

\subsection{Historique}

En phase opérationnelle depuis 7 ans, les travaux de conception et de prototypage de l'outil ont nécessité quatre années de travaux (cf. Figure 3), financées par l'ADEME-FC, la Région Franche-Comté, l'Agglomération du Grand 
Dole et l'Université de Franche-Comté (sous la forme d'autofinancement). Lors de cette première phase « recherche » du projet, ThéMA travaille sur le développement d'un prototype de plateforme internet d'observation et de prospective énergétique disposant d'outils de diffusion, de consultation et d'analyse de données à destination des décideurs locaux.

$\mathrm{Au}$ terme de cette première phase de conception-réalisation, l'association de surveillance de la qualité de l'air ATMO-FC se voit confier par les partenaires financeurs en 2010, le portage de l'outil. Ce choix est justifié du fait de la grande expérience d'ATMO-FC en matière de collecte, de gestion et d'analyse de données d'inventaires en vue de l'élaboration de diagnostics et de prévisions concernant la qualité de l'air. Il s'explique également par l'importance du réseau de partenaires fournisseurs de données avec lesquels ATMO-FC entretient des relations de confiance, ainsi que par les intérêts pour la structure de renforcer ses activités, audelà de la surveillance de la qualité de l'air, d'abord sur les problématiques climat dès 2006 puis sur l'énergie à partir de 2010.

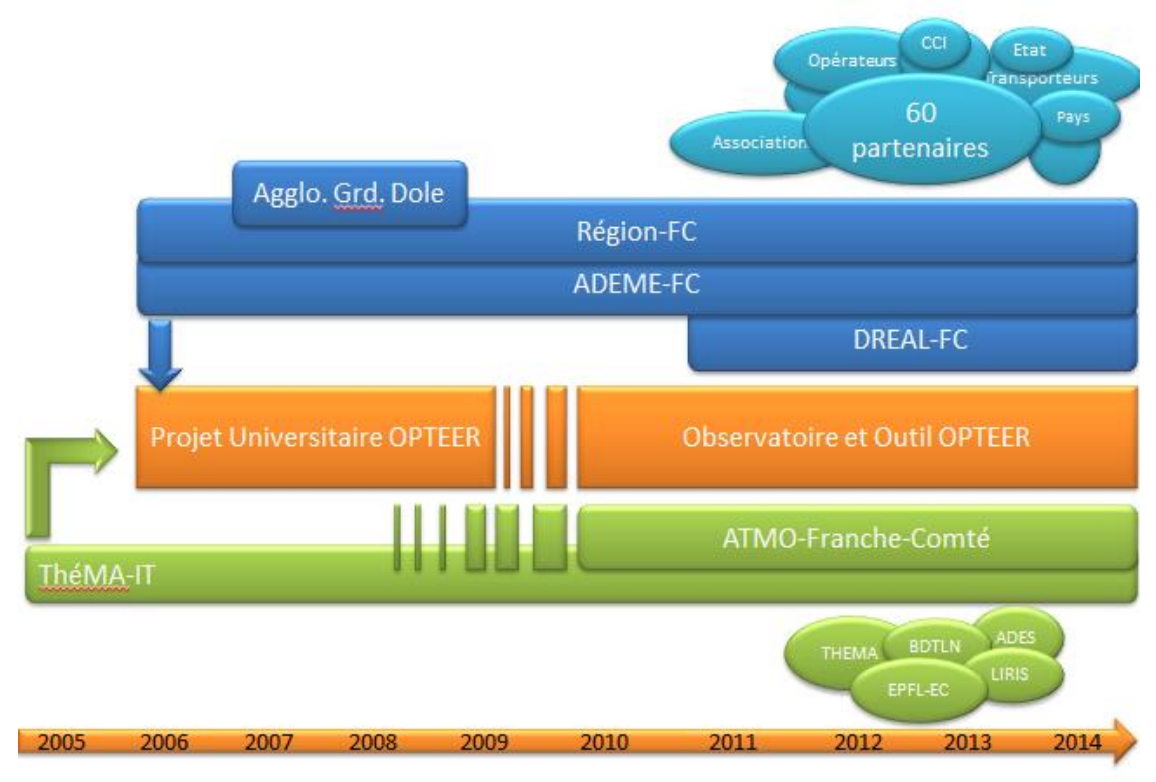

En vert, les partenaires scientifiques du projet, en bleu les partenaires institutionnels

Figure 3 : Historique de l'évolution du dispositif OPTEER

\subsection{Modes de gouvernance}

L'outil, développé au départ dans un cadre de recherche universitaire, a permis l'émergence puis la pérennisation d'une véritable dynamique socioorganisationnelle. Ainsi, depuis février 2010, un réseau d'acteurs locaux s'est 
organisé autour de l'outil OPTEER. Cette dynamique est assez atypique car on observe dans la plupart des Régions françaises la démarche inverse qui consiste préalablement à monter un réseau d'acteurs (appelé observatoire) autour de problématiques ciblées (logement, mobilité, précarité, ...) puis à chercher à développer un outil pour communiquer et diffuser des informations. Cette approche a d'ailleurs peut-être contribué à renforcer les échanges métiers et les liens inter partenaires autour de la finalité du dispositif et de ses intérêts, reléguant à l'arrièreplan les discussions et les problèmes «techniques » inhérents aux choix d'outils et de solutions techniques.

La gouvernance mise en place aujourd'hui autour de la plateforme s'articule autour des organes suivants (cf. figure 4) :

- Un organisme administrateur de l'outil et animateur du réseau, en l'occurrence ATMO Franche-Comté,

Un comité de pilotage regroupant l'ADEME, la Région et la DREAL de Franche-Comté (ayant rejoint le projet depuis la mise en place des SRCAE), le laboratoire ThéMA (Université de Franche-Comté) et ATMO Franche-Comté,

Un comité scientifique, composé de chercheurs français et suisses des domaines de l'informatique et de la géomatique, de l'énergie et de la géographie, dédié principalement à l'accompagnement scientifique et méthodologique nécessaire à l'évolution du système d'information OPTEER,

- $\quad$ Un comité technique qui regroupe l'ensemble des «partenaires fournisseurs de données » ainsi que les partenaires « réseau» (utilisateurs de la plateforme et experts thématiques). Certains membres de ce comité technique sont sollicités au besoin pour former des groupes de travail sur les données et indicateurs climat-airénergie,

- Des groupes de travail. Très rapidement après la mise à disposition de la plateforme auprès des acteurs de l'énergie en juin 2011, il est apparu nécessaire de s'appuyer sur les utilisateurs pour préciser collectivement les besoins en termes d'observation énergie-climat-air. Sept groupes de travail (GT) ont ainsi été définis. Il s'agit des GT «Bois-énergie », «Energies Renouvelables», «suivi des engagements », «Plans territoriaux», «Prospective», «Interopérabilité des outils », «stratégie ». Parmi ces groupes, les quatre premiers ont été lancés entre fin 2011 et début 2012. Ces GT ont réuni 44 acteurs régionaux provenant d'organismes très divers (collectivités, chambres consulaires, syndicat de l'énergie, agence d'urbanisme, parc naturel régional, université, Espaces Info Energie, ...). 


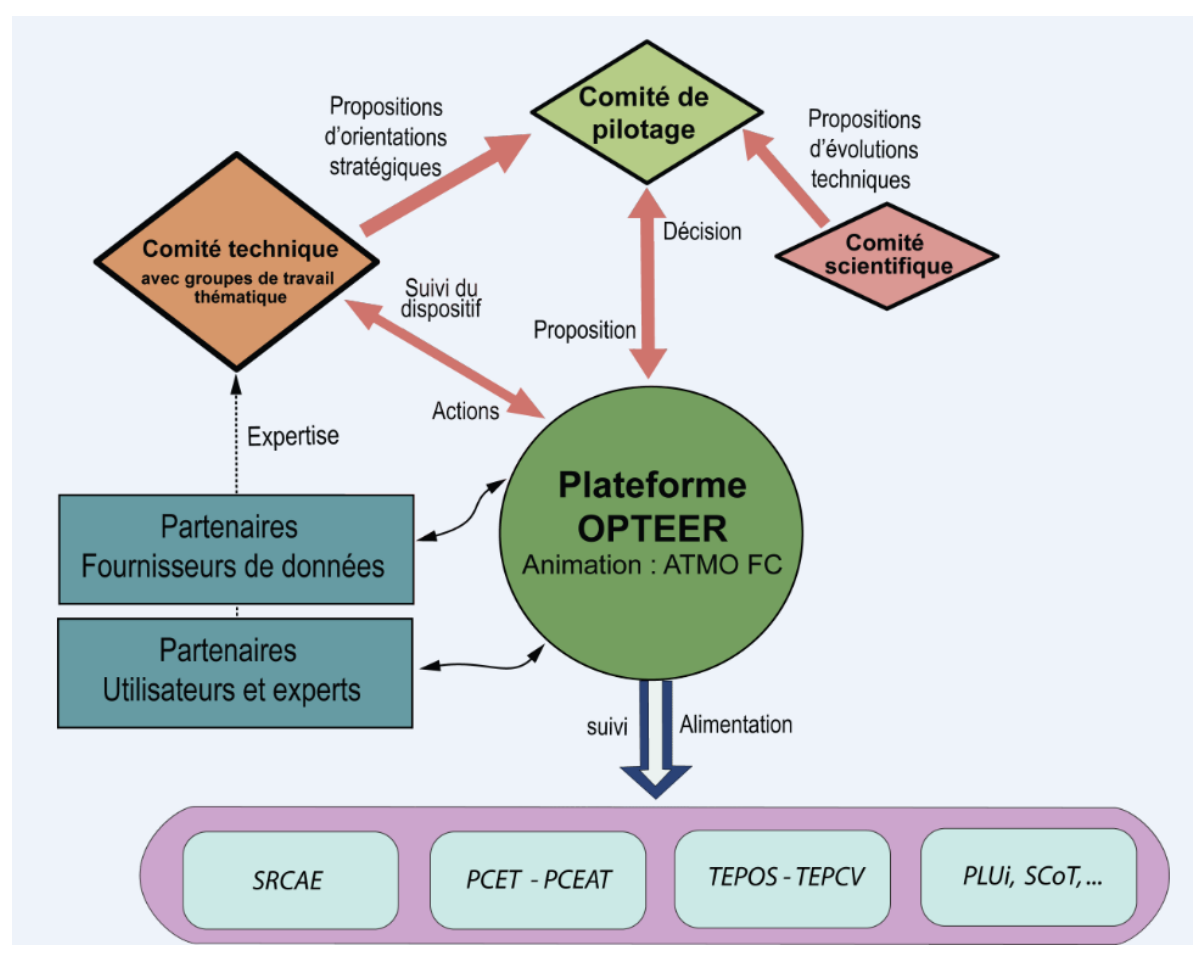

Figure 4 : Structure organisationnelle d'OPTEER

Le travail de consultation des acteurs locaux participant aux GT a permis à l'observatoire de progresser sur les questions portant sur la disponibilité des informations et les méthodes d'élaboration des indicateurs souhaités mais aussi sur les besoins en termes de qualité et de qualification des données et leur homogénéisation. Ainsi, environ 115 données, indicateurs et études ont été ciblés par les participants afin d'être mutualisés à terme et mis à disposition sur la plateforme OPTEER. Un important travail d'organisation des données et des indicateurs ciblés a été mené. Par ailleurs, une grille d'analyse a été élaborée afin d'évaluer le niveau de pertinence et de priorité des indicateurs définis (cf. Tableau 1).

Ces différents travaux menés en collaboration avec un large éventail d'acteurs de l'énergie ont permis la création d'indicateurs variés parmi lesquels un indicateur de vulnérabilité énergétique des ménages directement issu des réflexions du GT 2 pris en exemple dans le tableau 1 et la carte de la figure 5. Cet indicateur est construit sur la base d'une consommation énergétique globale des ménages (intégrant les dépenses énergétiques dans l'habitat et pour la mobilité) rapportée au revenu. Enfin, les acteurs ont également mis en avant l'intérêt de mettre à disposition des utilisateurs, et notamment des collectivités ne disposant pas des ressources 
suffisantes pour financer des compétences géomatiques, un ensemble de fiches territoires compilant des indicateurs énergétiques territoriaux pertinents, ainsi que des données de contexte / discriminants associés ou complémentaires pour l'analyse territoriale et attendus dans le cadre des démarches TEPOS par exemple.

\subsection{Données et choix techniques}

La plateforme OPTEER s'adresse en première intention à des acteurs locaux pour effectuer des études ainsi que des missions règlementaires sur leur territoire. Elle a vocation à fournir, présenter et permettre l'analyse de données et la production d'indicateurs à ses utilisateurs. Pour ces raisons, la plateforme a été pensée pour répondre aux exigences qu'impliquent le suivi, le traitement et la représentation/diffusion d'informations spatio-temporelles multi-échelles.

Pour ce qui concerne les données (cf. figure 6), il va sans dire que celles-ci doivent être fiables, pérennes et suffisamment détaillées pour permettre la description de la situation énergétique, climatique et environnementale actuelle et de son évolution sur les territoires concernés et répondre aux besoins exprimés par les partenaires réunis autour de la plateforme (cf. tableau 1)

L'énergie est produite par l'intermédiaire de divers processus (combustion, géothermie, fission nucléaire etc.), à partir de différentes ressources (vecteurs primaires (bois, uranium, charbon, soleil, ...)). Elle est par ailleurs utilisée pour de multiples usages (force motrice, chauffage, transport, ...) dans l'ensemble des secteurs d'activité (industriel, résidentiel, ...). Cette complexité explique que les données se rapportant à l'énergie relèvent, en France, de nombreux services au sein de différentes structures. La disponibilité des données portant sur cette thématique au sens large, est donc marquée par le caractère hétérogène et dispersé de l'information, les données énergétiques, pouvant porter sur les productions, les consommations, les usages voire les comportements. 
Tableau 1. Exemple de document de travail mis en place dans le cadre des GT

GT2 : "Plans territoriaux », liste des thèmes et des indicateurs

Ce document a été complété avec l'ensemble des membres du groupe de travail lors de la séance du 29.11.2011

\begin{tabular}{|c|c|c|c|c|c|c|}
\hline $\begin{array}{l}\text { Indicateurs } \\
\text { chapeau }\end{array}$ & $\begin{array}{c}\text { Indicateurs } \\
\text { détaillé par } \\
\text { Problématique }\end{array}$ & Thématiques & Acteurs & $\begin{array}{l}\text { Exemple de données } \\
\text { ou indicateurs }\end{array}$ & $\begin{array}{c}\text { Difficulté } \\
\text { (de collecte ou } \\
\text { de création) }\end{array}$ & $\begin{array}{l}\begin{array}{c}\text { Niveau } \\
\text { de } \\
\text { priorité }\end{array} \\
\mathbf{1} 203 \\
\text { Elevé } \\
\text { Moyen } \\
\text { Faible } \\
\end{array}$ \\
\hline \multirow{2}{*}{$\begin{array}{c}\text { Vulnérabilité } \\
\text { énergétiques } \\
1 \text { - Logement } \\
\text { 2- Transport } \\
\text { 3- Economie locale- } \\
\text { agriculture } \\
\text { 4- Urbanisme } \\
\text { 5- Comportement? } \\
6 \text { - Energie }\end{array}$} & $\begin{array}{c}\text { 1- Logement } \\
\text { Avec } \\
\text { Précarité énergétique }\end{array}$ & $\begin{array}{l}\text { - Mobilité } \\
\text { - Socio-économie } \\
\text { - Géographie } \\
\text { - Urbanisme } \\
\text { - Energie }\end{array}$ & $\begin{array}{l}\text { - ThéMA (travaux en cours) } \\
\text { ORT Franche-Comté } \\
\text { - INSEE } \\
\text { Agences d'urbanisme } \rightarrow \text { observatoire départemental de } \\
\text { l'Habitat (publication sur le sujet) - } \\
\text { - ADIL } \\
\text { - EIE } \\
\text { - CG } \\
\text { CASS } \\
\text { - ARS } \\
\text { - Fournisseurs d'énergie (EDF, GDF-Suez) } \\
\text { - AJENA (des travauu vont être lancés sur le sujet) }\end{array}$ & $\begin{array}{l}\text { - Indicateur traduisant le taux } \\
\text { de ménagesen situation de } \\
\text { précarité énergétique } \\
\text { - Les différents indicateurs } \\
\text { intermédiaires (socio- } \\
\text { économie, mobilité,...) clef } \\
\text { d'analyse entre urbain et rural } \\
\text { - Nombre de ménages aidés via } \\
\text { les } \mathrm{CG}, . . .\end{array}$ & - & (1) \\
\hline & $\begin{array}{l}\text { 1- Résidentiel collectif } \\
\text { Logements sociaux } \\
\text { + logements } \\
\text { individuels }\end{array}$ & $\begin{array}{l}\text { - Bâtiment } \\
\text { - Socio-économie } \\
\text { - Energie }\end{array}$ & $\begin{array}{l}\text { - Bailleurs sociaux } \\
\text { - DREAL } \\
\text { - Union sociale pour I'habitat (USH) } \\
\text { - Caisse des Dépôts } \\
\text { - Conseils Généraux } \\
\text { ADIL } \\
\text { - EIE } \\
\text { - Collectivités (aides à la pierre) } \\
\text { - Rénovation urbaine » }\end{array}$ & $\begin{array}{l}\text { - Taux d'amélioration } \\
\text { énergétique dans le logement } \\
\text { social ( (quelle définition pour } \\
\text { " amélioration »?) } \\
\text { - Indicateur sur le cout global } \\
\text { (BBC) (Difficilement } \\
\text { intégrable dans OPTEER) } \\
\text { - Nombre de fond de solidarité } \\
\text { des logements (FSL) délivrés }\end{array}$ & & 2 \\
\hline
\end{tabular}

Nom entier de la revue $-\mathrm{n}^{\circ}$ 1/2017, 1-5 AR_pied-page1 


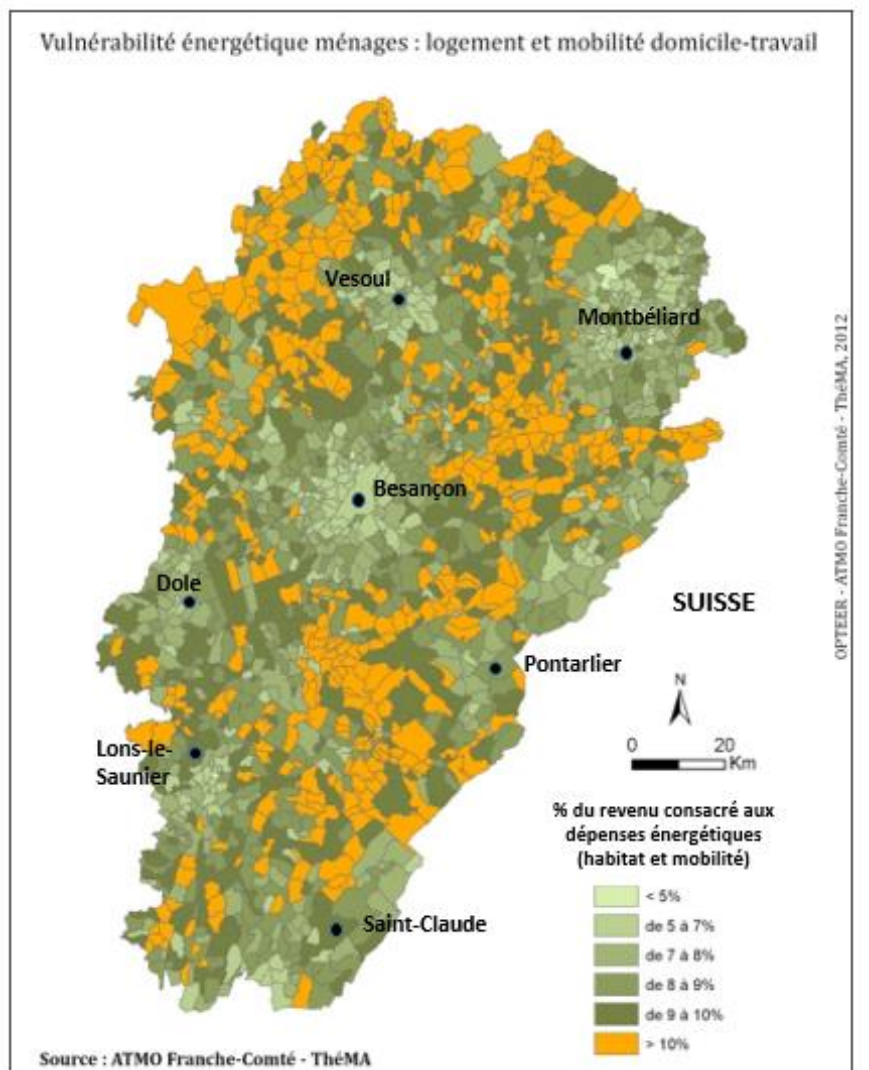

Figure 5: Un exemple de production d'un indicateur composite, issu des travaux des GT

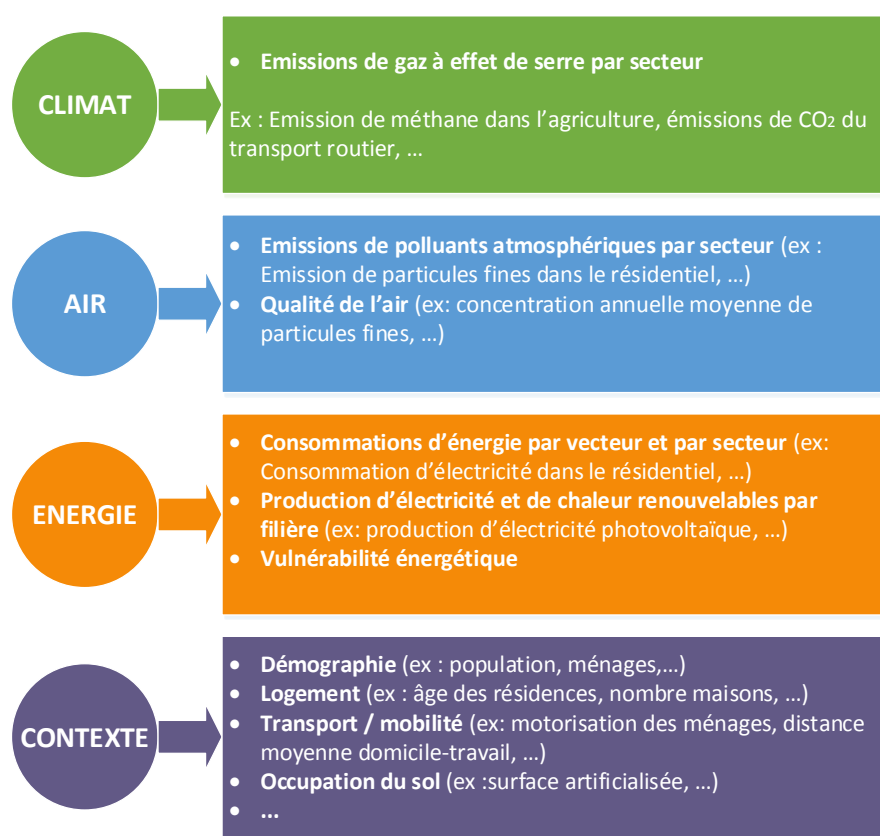

Figure 6 : Vision synthétique des données disponibles au seir plateforme 
Le nombre de sources de données reste toutefois relativement limité, si l'on compare la situation française à celle de certains autres pays telle que la Suisse (850 entreprises d'approvisionnement en électricité). A titre d'exemple, on compte en Franche-Comté, sept organismes fournisseurs de données relatives à l'approvisionnement en électricité et en gaz de ville ${ }^{13}$. Pour autant, l'accès aux informations s'avère souvent compliqué pour les collectivités, malgré leur légitimité (par exemple en tant que structures organisatrices pour l'électricité) à disposer des données les concernant. Cependant, il est à noter que les évolutions réglementaires dans le cadre de la loi pour la Transition Energétique et la Croissance Verte (article 179 de la Loi TECV sur les données énergétiques) vont dans le sens d'un meilleur accès aux données ${ }^{14}$.

Par ailleurs, l'élaboration d'inventaires territoriaux nécessite la mise en place de campagnes de collecte de données à l'échelle régionale, à l'image de celles réalisées par ATMO-FC depuis 2005, pour la construction de l'inventaire franc-comtois des émissions de polluants et de GES (Gaz à Effet de Serre). La majeure partie de l'évaluation des émissions de polluants et de GES réalisée par ATMO-FC, débute par un diagnostic des consommations énergétiques pour tous les secteurs d'activités de la région. ATMO-FC dispose de méthodologies et d'outils développés au sein des AASQA (Associations Agréées pour la Surveillance de la Qualité de l'Air) sur la base de travaux faisant référence en France comme en Europe. Ceux-ci permettent de traduire, en émissions de GES et de polluants, les indicateurs d'activités telles que les consommations d'énergie à l'échelle communale. Afin de compléter ces inventaires, ATMO-FC contractualise également avec certains gestionnaires de réseaux qui fournissent des données sur les consommations de gaz et d'électricité ainsi que sur certains modes de production d'énergie. Mentionnons enfin le recours à un modèle de trafic pour alimenter la plateforme en données sur la mobilité à des échelles fines, ainsi que le modèle de consommation / émission basé sur la méthodologie COPERT.

La collecte des données et la mise en œuvre des méthodes permettant la construction d'inventaires territoriaux climat-air-énergie à échelle fine sont chronophages mais nécessaires pour proposer aux différentes collectivités une base de données unique, fiable et la plus exhaustive possible.

D'un point de vue technique, considérant les exigences en termes de complexité, de volumétrie, de sécurisation et de diffusion des données, les choix se sont orientés vers une architecure basée sur MapServer, Serveur cartographique compatible OGC conforme INSPIRE, avec sa suite logiciel MapCache permettant notamment d'accélérer l'accès aux couches WMS de l'OGC (figure 7).

\footnotetext{
${ }^{13}$ RTE - Enedis - SICAE- SIEL- Régie de Salin les bains - GRT - GrDF

14 "Deux décrets et un arrêté publiés le 20 juillet au Journal officiel prévoient de donner aux collectivités locales un accès gratuit à des données sur la production et la consommation d'énergie sur leur territoire. Un outil de gestion utile à l'élaboration des schémas régionaux et des Plans climat-air-énergie territoriaux, même si l'accès aux données se fera progressivement de 2016 à 2021 ». Source : http://www.courrierdesmaires.fr/63497
} 


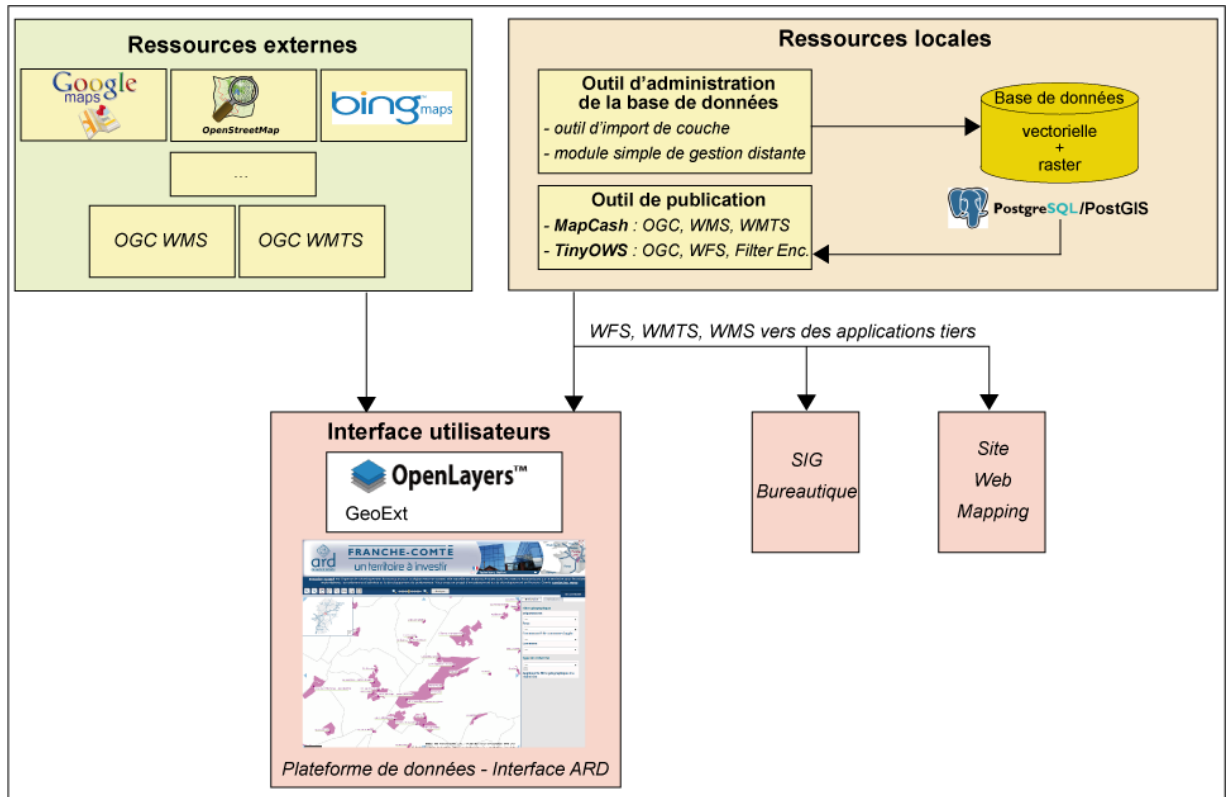

Figure 7 : Architecture de l'outil OPTEER, (source : IAD Territoire Digital, 2014)

La solution d'entreposage de données retenue permet aux utilisateurs, au-delà de la consultation, de faire de la gestion et de l'administration des données qui le composent.

L'ensemble de ces choix permettent la mise à disposition des professionnels des éléments suivants :

- La qualification et l'importation autonome, par des utilisateurs noninformaticiens (chargés d'études statistiques par exemple), de données hétérogènes ;

- L'hébergement sécurisé (selon les recommandations de la CNIL);

- Le requêtage sur les données brutes, la création et les sauvegardes de requêtes et d'indicateurs mêlant données et formules par des utilisateurs noninformaticiens ;

- La construction de territoires et d'indicateurs personnalisés ;

- La diffusion des données et des indicateurs à travers leur description (administrable via un contrôle de textes enrichis) et la visualisation de cartographies thématiques en ligne

- La création, sauvegarde et diffusion de profils de territoire dynamiques exportables comportant des tableaux, des cartes et des graphiques 
d'évolution (aux formats .PDF, .SVG, .XLS ou .DOC). Ces profils de territoire, dont le contenu est déterminé pour chaque niveau, peuvent être multipliés sur un même de territoire (généraliste, thématiques...) (figures 8a, $8 \mathrm{~b}$ et $8 \mathrm{c})$;

- La fourniture et la mise à jour de fonds de cartes et de zonages géographiques métier (type : Départements, Zones d'emploi, EPCI, Communes, ...) ;

- L'administration des profils d'utilisateurs, des comptes utilisateurs, des zonages géographiques personnalisés, des données, des formules et des illustrations (cartes, graphiques et tableau).

- La mise à disposition d'un portail grand public (figure 9) avec un accès sécurisé aux utilisateurs experts (ils bénéficient de fonctionnalités avancées et plus complètes que le grand public);

\subsection{Dispositifs d'animation et d'accompagnement}

L'animation et l'accompagnement des utilisateurs de la plateforme s'articulent autour de différentes actions complémentaires.

ATMO-FC assure l'animation du comité de pilotage de l'observatoire, du comité technique et d'un réseau d'acteurs territoriaux rassemblant en 2016 plus de 60 organismes, dont les utilisateurs de la plateforme OPTEER et les fournisseurs de données. Cette dynamique s'explique notamment en réponse aux besoins liés à l'élaboration et au suivi de l'ensemble des schémas et autres plans en lien avec la triple thématique air énergie climat (SRCAE, PCET, PCEAT, TEPOS, SCoT (Schéma de Cohérence Territoriale) ...). Cet accompagnement des territoires depuis 2010, nous amène aujourd'hui au constat d'une réelle appropriation des données et des outils de la plateforme OPTEER s'accompagnant d'une montée en compétence des chargés de mission qui œuvrent à l'élaboration des plans et schémas divers. La pertinence de leurs remarques et les demandes de plus en plus exigeantes qu'ils formulent apparaissent comme des éléments moteurs du processus d'amélioration continue à l'œuvre.

Les réflexions autour de l'évolution de l'ensemble du dispositif se font par le biais de l'animation des comités techniques et groupes de travail regroupant experts dans les thématiques en lien avec l'observatoire, utilisateurs (collectivités territoriales, services de l'Etat, agences, etc.) et producteurs de données (les énergéticiens, les filières Bois Energie, etc.). 


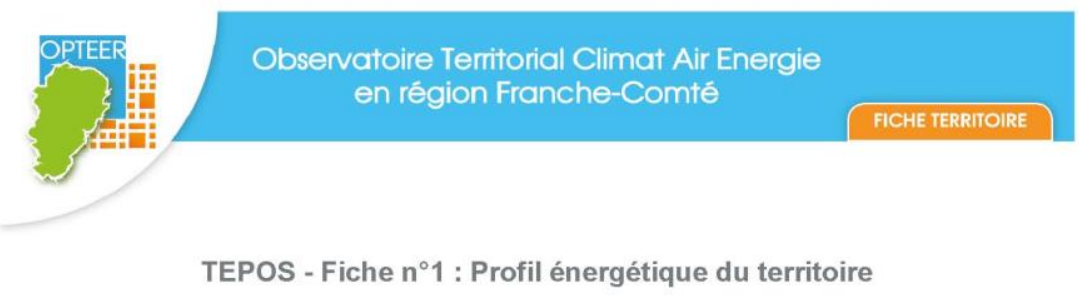

"Un territoire à énergie positive vise l'objectif de réduire ses besoins d'énergie au maximum, par la sobriété et l'efficacité énergétiques, et de les couvrir par les énergies renouvelables locales ("100\% renouvelables et plus")". unw.ternitoires-energie-positive.fr

Cette fiche regroupe un ensemble d'indicateurs généraux permettant de dégager les principaux enjeux des territoires engagés dans la démarche TEPOS. Elle apporte également des éléments sur les impacts climatique, environnemental, et des indicateurs dynamiques de suivi de la trajectoire du territoire vers un "Territoire à Energie POSitive".

Territoire personnalisé : Mon territoire de projet TEPOS

\section{Contexte}

Population

\begin{tabular}{|c|c|c|c|}
\hline & & $\begin{array}{r}\text { Mon temitione de propet } \\
\text { TEPOS }\end{array}$ & Franche-Comte \\
\hline $\begin{array}{l}\text { Population municipale } \\
\text { (habitant(s)) } \\
\text { Source : INSEE }\end{array}$ & 2014 & 19350 & 1178003 \\
\hline $\begin{array}{l}\text { Densité de population } \\
\text { (Hab/km2) } \\
\text { Source : INSEE }\end{array}$ & 2014 & 27,60 & 72,40 \\
\hline
\end{tabular}

Figure 8 a : Un exemple de production à destination des collectivités ; extraits de la fiche TEPOS 


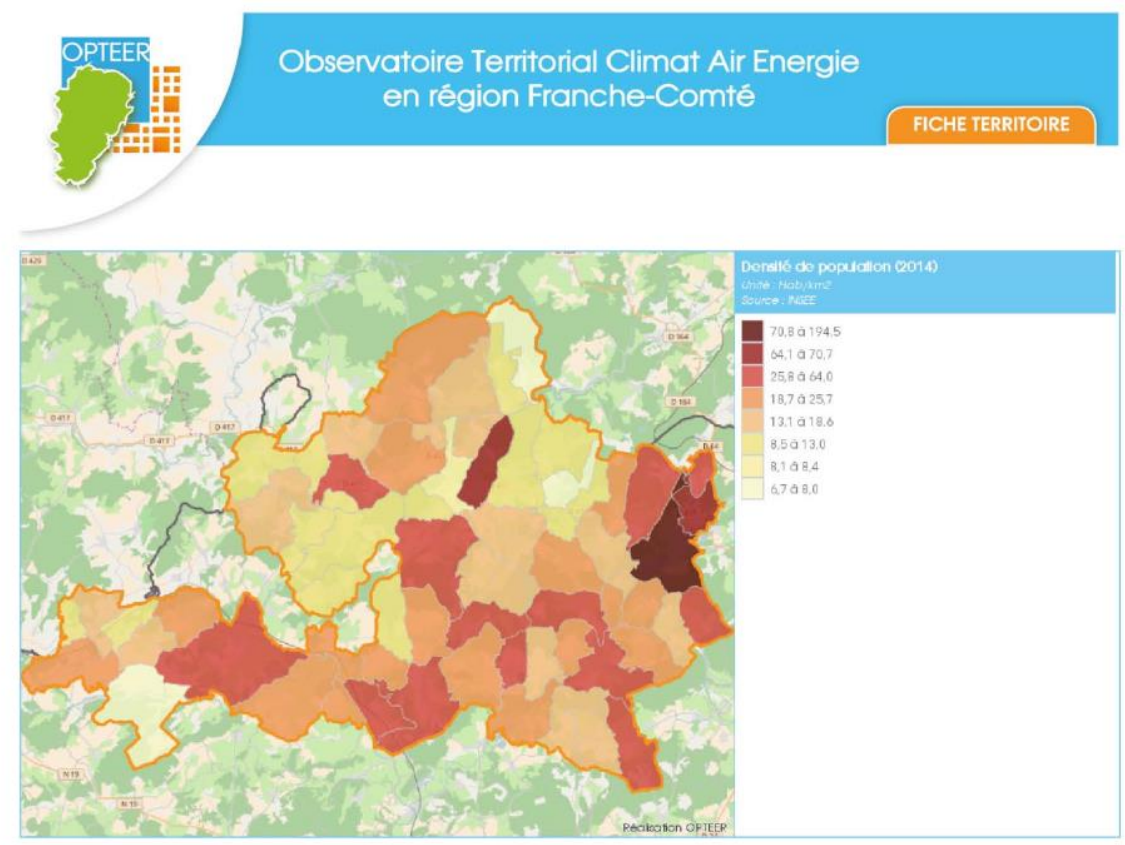

Le graphique ci-dessous permet d'observer les évolutions démographiques sur votre territoire depuis 1962. La donnée utilisée porte sur la population municipale (ex-population sans doubles comptes) issue du recensement de la population réalisé par l'INSEE.

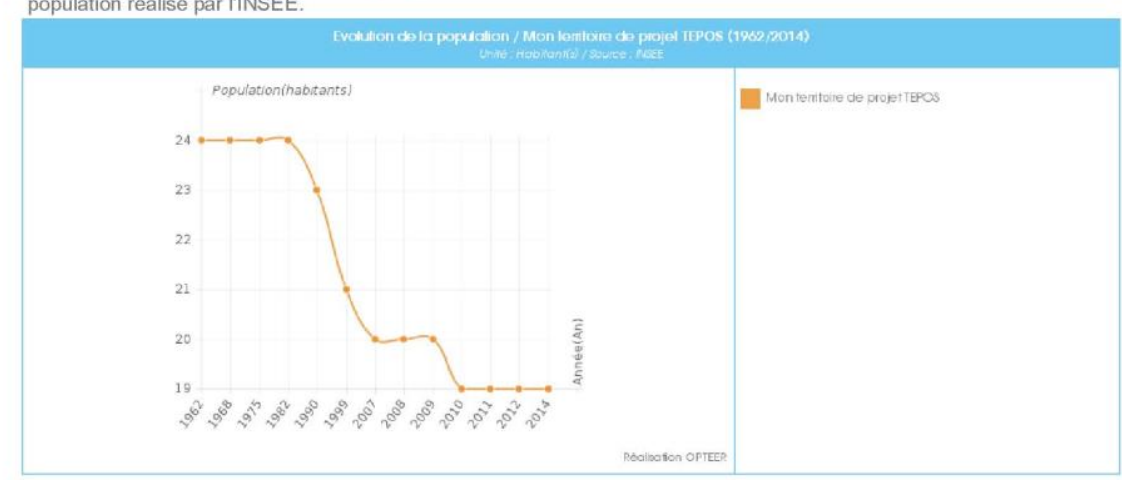

Emplois

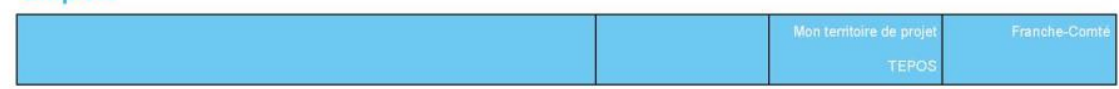

$1505 / 2017$

Figure $8 \mathrm{~b}$ : Un exemple de production à destination des collectivités; extraits de la fiche TEPOS 

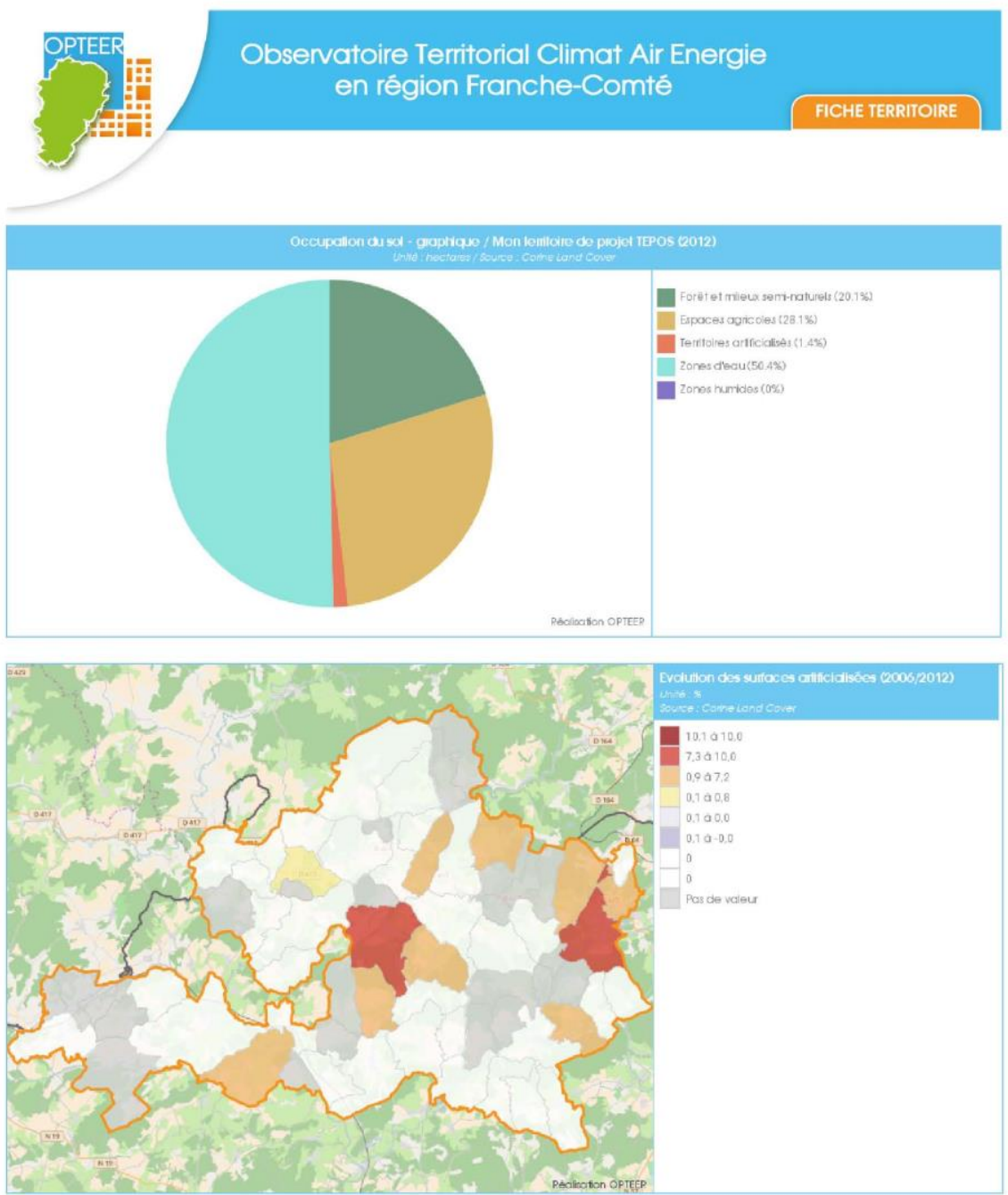

Indicateurs de trajectoire TEPOS

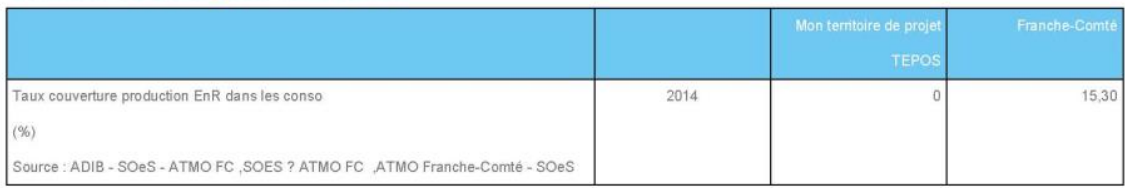

$15 / 05 / 2017$

Figure $8 \mathrm{c}$ : Un exemple de production à destination des collectivités ; extraits de la fiche TEPOS 


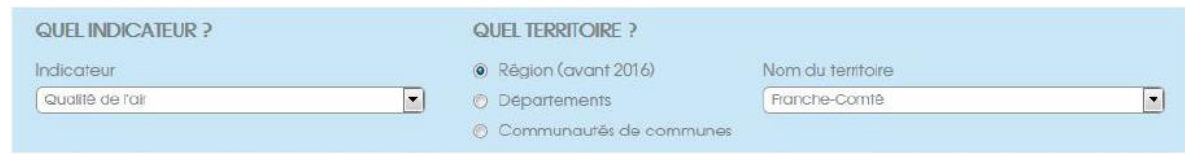

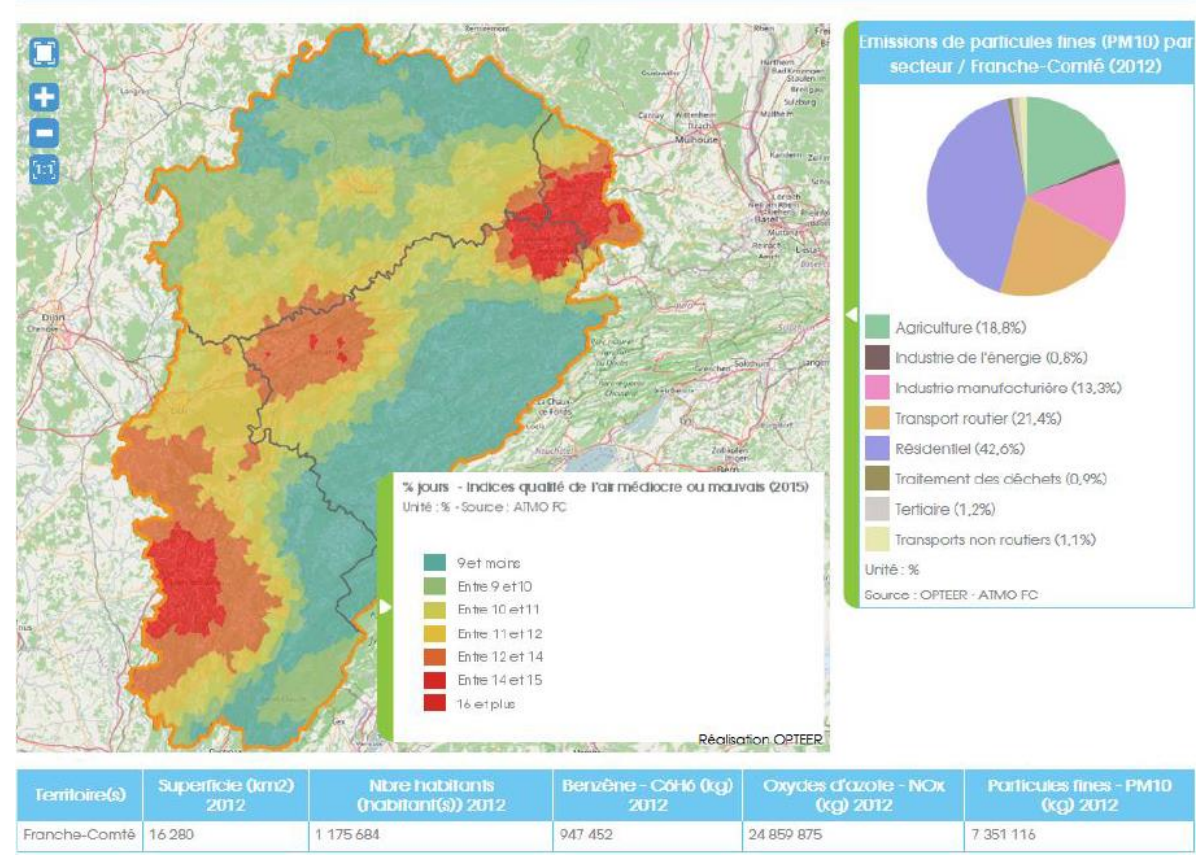

Figure 9 : Exemple de production de la plateforme à destination du grand public

Enfin, des relations étroites sont maintenues avec les milieux scientifiques, dans le cadre d'une participation au comité scientifique d'OPTEER piloté par le Laboratoire ThéMA de l'Université de Franche-Comté (initiateur du projet et propriétaire de l'outil). Cette action permet, d'une part, de maintenir un caractère innovant à ce système d'information territorial, et, d'autre part, de collaborer activement aux réflexions menées sur les approches territoriales de l'énergie et aux travaux dans les domaines de la géomatique et des Systèmes d'Information. 


\section{Bilan, analyse et perspectives d'évolution du dispositif OPTEER}

\subsection{Bilan et analyse des pratiques et des usages autour de l'outil}

\subsubsection{Bilan des activités à $T+5$}

Depuis 2010, on constate une croissance constante du nombre d'utilisateurs de l'outil et de la fréquentation de la plateforme (figure 10). Plus précisément en 2016 , 12689 opérations ont été réalisées, avec 4558 cartes ou graphiques consultés et 6327 données exportées. Ces chiffres correspondent à 55 opérations par jour en moyenne. 41 nouveaux utilisateurs ont été recensés pour cette même année et 16 organismes ont demandé des comptes d'accès à la plateforme.

En termes d'accompagnement, plus de 90 personnes ont été formées à l'utilisation d'OPTEER en formation initiale. 6 journées de formation ont été organisées en 2016 dont 4 spécifiquement dans le cadre de la mise en œuvre des TEPOS pour une participation évaluée à 33 personnes.

ATMO-FC évalue à 6 jours/homme/mois l'accompagnement du dispositif, qu'il s'agisse d'assistance, d'accompagnement à la compréhension et l'analyse des indicateurs fournis ou à la construction ou de la mise à disposition de données complémentaires spécifiques suite à des demandes d'utilisateurs.

Enfin, en termes de bilan, il est intéressant de se pencher sur les types de contenu les plus consultés et les plus utilisés en 2016. La figure 11 nous montre la prééminence de la consultation des données énergétiques alors que la majorité des données exportées sont des données dites de contextes (données sociodémographiques par exemple). Cet état de fait peut s'expliquer par le rôle d'infrastructure de données joué par la plateforme OPTEER, en l'absence d'un dispositif de ce type à l'échelle régionale.

\subsubsection{Analyse des usages de l'outil}

Les usages de la plateforme OPTEER sont très liés aux évolutions réglementaires et aux attentes en matière de documents de planification dans le cadre de la loi sur la Transition Energétique. Dans ce contexte, la diversité des projets et des porteurs est à noter avec notamment une augmentation des usages dans le cadre du SRCAE porté par la Région et la DREAL, des PCET avec les Pays et les EPCI, des démarches Cit 'ergie / convention des Maires, des PLUi-PDU-SCoT, PCAET avec les communes et agglomérations mais aussi réalisés par des bureaux d'études ou des agences d'urbanisme, des TEPOS-TEPCV, portés quant à eux par les EPCI (Etablissement Public de Coopération Intercommunale), le plus souvent sur le périmètre d'un pays (figure 12). Dans une démarche d'amélioration continue, des échanges réguliers entre les utilisateurs et l'animateur de l'observatoire ont lieu afin de suivre l'évolution des besoins et d'anticiper les réponses à apporter. Sans que cela ne se fasse par l'intermédiaire d'un questionnaire, le niveau de satisfaction des usagers de l'outil est constamment évalué à travers ces échanges. La plateforme 
révisée en 2015, intégrant l'ensemble des remarques des utilisateurs depuis 2010, répond à ce jour aux besoins des territoires dans le cadre de l'élaboration de leur politiques locales Climat-Air-Energie.

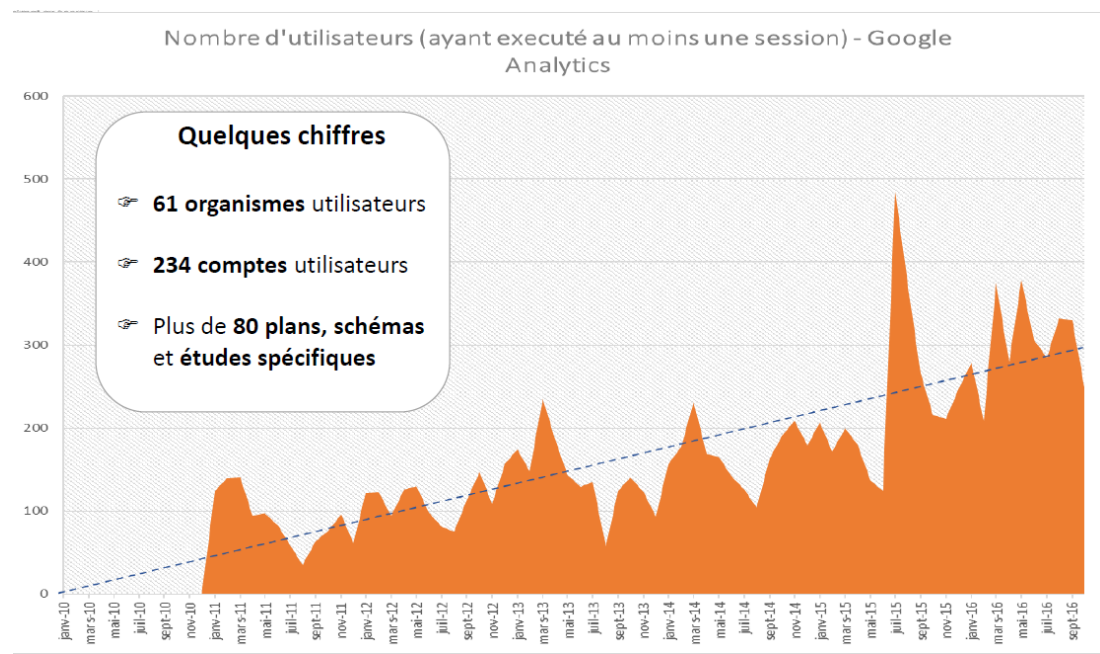

Figure 10 : Utilisation de la plateforme depuis 2010

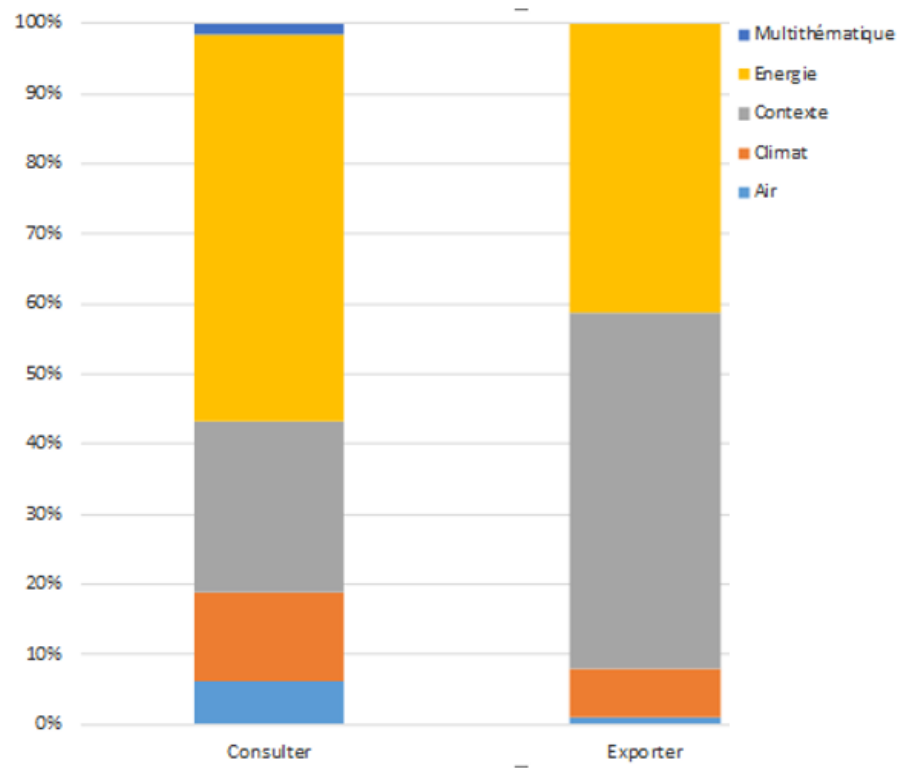

Figure 11 : Champs thématiques de données consultées et exportées en 2016 
Ces évolutions contribuent à la grande diversité des utilisateurs de la plateforme constatée en 2016 (Cf. tableau 2). Aujourd'hui, plus d'une soixantaine d'organismes (collectivités territoriales, associations, services déconcentrés de l'État, chambres consulaires, université, transporteurs ou fournisseurs d'énergie, bureaux d'études...) sont impliqués dans les actions de l'observatoire OPTEER ou sont concernés par celles-ci.

Mais au-delà de cette typologie des utilisateurs, l'accompagnement et l'animation qui caractérisent le dispositif OPTEER nous permettent d'aller plus loin dans la compréhension des usages des productions de la plateforme. Le recueil des besoins des partenaires, notamment lors des séances des différents groupes de travail a permis la co-construction d'indicateurs mis ensuite à la disposition de tous. A titre d'exemple nous pouvons citer des indicateurs de vulnérabilité énergétique des ménages, de distance moyenne domicile-travail, de consommation énergétique et d'émissions sur les autoroutes, ... Des modèles de fiches pour les TEPOS ont été également développés avec l'ADEME, la Région et la DREAL, avec notamment la production des données d'entrée (nombre d'emploi, km parcours par motifs, consommations d'énergie du transport, des bâtiments, ...) nécessaires à l'utilisation d'un outil de simulation développé par la Région (boite à outils TEPOS) et utilisables par les territoires, peu importe leur taille. Cette fiche dynamique disponible sur OPTEER permet de produire rapidement des chiffres clefs recalculés automatiquement quelles que soient les échelles et les dates.

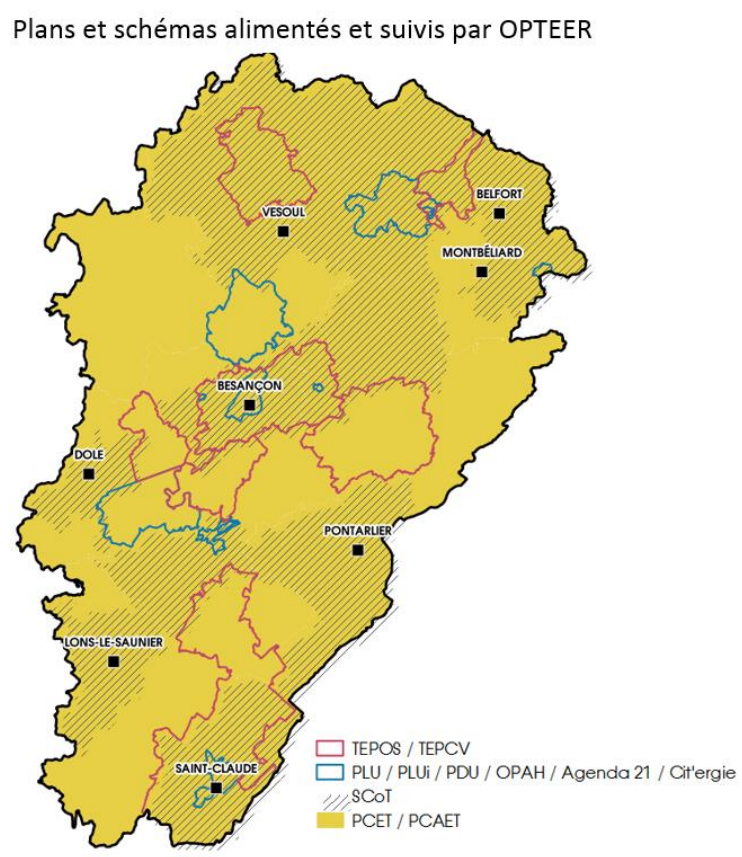

Figure 12 : Usages de la plateforme en Franche-Comté dans le cadre de la constitution de documents d'urbanisme et de planification 
Ainsi, depuis 2010, OPTEER a contribué à l'élaboration de tous les plans et schémas stratégiques territoriaux portant sur l'énergie, le climat et l'air (plus de 80 plans, schémas ou études spécifiques) en Franche-Comté.

Tableau 2. Organismes utilisateurs d'OPTEER à la fin 2016

\begin{tabular}{|c|c|c|}
\hline Collectivités territoriales & Bureaux d'études & Parcs Naturel Régionaux \\
\hline CAGB - Communauté d'agglomération du Grand-Besançon & AERE & PNR Doubs \\
\hline CAGD - Communauté d'agglomération du Grand Dole & Alter Eco & PNR Haut-Jura \\
\hline Communauté de communes de Champagnole Porte du Haut-Jura & AXENNE & Université, laboratoires ou instituts de \\
\hline Communauté de communes des Terres de Saône & ENERGIO & recherche \\
\hline Communauté de communes du Canton de Quingey & EXPLICIT & Science Po - Paris \\
\hline Communauté de communes du Haut-Jura - Arcade & Hêlianthe & Université Bourgogne-Franche-Comté \\
\hline Communauté de communes Haut-Jura Saint-Claude & INDDIGO & Association Négawatt \\
\hline Communauté de communes Jura Nord & Initiative A et D & Services de l'Etat \\
\hline Communauté de communes Jura Sud & Kovalence & ADEME Bourgogne - Franche-Comté \\
\hline $\begin{array}{l}\text { Conseil départemental du Doubs } \\
\text { Conseil départemental du Jura }\end{array}$ & $\begin{array}{l}\text { Rovalence } \\
\text { Mosaique Environnement } \\
\end{array}$ & Chambre régionale d'agriculture Franche-Comté \\
\hline Conseil départemental du Territoire de Belfort & SOBERCO & DREAL Bourgogne - Franche-Comté \\
\hline Conseil dèpartemental de Haute-Saône & \multicolumn{2}{|r|}{ Agences et syndicats } \\
\hline Conseil Régional Bourgogne-Franche-Comté & \multicolumn{2}{|c|}{ ADU - Agence d'urbanisme de l'agglomération de Montbéliard } \\
\hline ECLA - Espace Communautaire Lons Agglomération & \multicolumn{2}{|c|}{ AUDAB - Agence d'urbanisme de l'agglomération bisontine } \\
\hline Pays des 7 Rivières & \multicolumn{2}{|c|}{ AUTB - Agence d'urbanisme du Territoire de Belfort } \\
\hline Pays des Portes du Haut-Doubs & \multicolumn{2}{|c|}{ HDL - Habitat Développement local du Doubs et du Territoire de Blefort } \\
\hline $\begin{array}{l}\text { Pays des Vosges Saônoises } \\
\text { Pays Dolois }\end{array}$ & \multicolumn{2}{|l|}{ Institut Négawatt } \\
\hline Pays du Doubs Central & \multicolumn{2}{|c|}{ SMAU - Syndicat Mixte de l'Aire Urbaine Belfort-Montbéliard (PCAET de l'AUBM) } \\
\hline Pays du Revermont & \multicolumn{2}{|c|}{ SYDED - Syndicat de Mixte d'électricité du Doubs } \\
\hline Pays Graylois & \multicolumn{2}{|c|}{ SIED 70 - Syndicat intercommunal d'énergie de Haute-Saône } \\
\hline Pays Horloger & \multicolumn{2}{|c|}{ SIDEC - Syndicat Mixte d'énergie du Jura } \\
\hline Pays Loue Lison & \multicolumn{2}{|c|}{ Associations et Espaces info énergie } \\
\hline $\begin{array}{l}\text { Pays Vesoul - Val de Saône } \\
\text { PMA - Pays Montbéliard Agglomération }\end{array}$ & \multicolumn{2}{|c|}{$\begin{array}{l}\text { ADERA } 70 \text { - Association pour le développement des énergies renouvelables et } \\
\text { alternatives en Haute-Saône et EIE }\end{array}$} \\
\hline SMAU - Syndicat Mixte de I'Aire Urbaine Belfort-Montbéliard (élaboration du & \multicolumn{2}{|c|}{ AJENA - Energie et environnement en Bourgogne-Franche-Comté et EIE } \\
\hline PCAET de I'AUBM) & \multicolumn{2}{|c|}{ Gaìa Energies - Association pour la maitrise de l'énergie - territoire de Belfort et EIE } \\
\hline $\begin{array}{l}\text { Ville de Besançon } \\
\text { Ville de Delle }\end{array}$ & \multicolumn{2}{|c|}{ Observatoire Régional de la Santé Bourgogne - Franche-Comté } \\
\hline $\begin{array}{l}\text { Ville de Delle } \\
\text { Ville de Montbéliard }\end{array}$ & \multicolumn{2}{|c|}{ URACoFor - Union régionale des communes forestières } \\
\hline Ville de Saint-Claude & \multicolumn{2}{|c|}{ ADIB - Association des industriels du bois } \\
\hline
\end{tabular}

\subsection{Le dispositif OPTEER dans le paysage des observatoires/SI environnement climat.}

OPTEER s'inscrit dans un réseau d'observatoires européen. Par sa contribution au programme ClimactRegions ${ }^{15}$ en 2011 , il fait partie des premiers dispositifs d'observation ayant rejoint le réseau européen ENERGee-Watch, avant même son ouverture officielle ${ }^{16}$ et au sein duquel il est un membre actif. Cet engagement se traduit par des échanges réguliers se déroulant plusieurs fois par an (webinars, ...). Les travaux portent notamment sur la définition d'indicateurs communs entre tous les observatoires régionaux européens membres d'ENERGee-Watch. Cette participation est importante et permet une veille nécessaire sur les évolutions du domaine de l'observation énergétique.

\footnotetext{
$15 \mathrm{http}: / /$ www.climactregions.eu/web/guest/project

${ }^{16} \mathrm{http}: / /$ www.energee-watch.eu réseau européen des observatoires régionaux de l'énergie et des GES porté par la fédération européenne des régions et des agences pour l'énergie et l'environnement (FEDARENE).
} 
Différentes études comparatives ont été menées en France sur les dispositifs d'observation Air Energie Climat. Les conclusions de ces travaux confirment la variété des solutions mises en œuvre selon les régions notamment en termes de modes de gouvernance. L'examen de ces études montre notamment (Chabret, 2014) la diversité des fonctionnalités offertes par le dispositif OPTEER, l'importance accordée à la qualité et à la sécurisation des données et l'originalité du mode de gouvernance mis en place.

Enfin, selon (Mazaud et al., 2017), les outils à vocation territoriale qui produisent de l'information à destination des décideurs peuvent se décliner selon deux grandes familles: les outils de capitalisation de données et les outils de modélisation. Où positionner OPTEER dans ce cadre d'analyse ? OPTEER est tout d'abord un dispositif de capitalisation active de données variées, à différents niveaux d'échelle, en complémentarité avec les IDG (Infrastructures de Données Géographiques). Au-delà de la capitalisation, les capacités d'analyse spatiotemporelle alimentées par des données multithématiques et adossées à des compétences d'experts contribuent à la production d'une véritable connaissance territoriale. Tous ces éléments démontrent ainsi, via l'important travail de synthèse et les efforts investis dans la communication d'indicateurs territoriaux composites, la dimension d'outil de connaissance que la plateforme a pris pour les territoires. Ainsi en est-il des tableaux de bord, fiches territoires et autres productions spécifiques telles que les fiches TEPOS à destination des collectivités. Enfin, cette approche est confortée par le couplage de l'outil avec la chaine de modélisation des données Climat-Air-Energie d'ATMO-FC comprenant, entre autre, un modèle de trafic exploité pour l'analyse. Ce couplage observation-modélisation devrait demain permettre de répondre aux attentes des partenaires en matière de prospective et de scénarisation.

\subsection{Nouveaux besoins autour des approches énergie/climat/environnement à l'échelle des territoires et importance des liens "recherche et niveau opérationnel ».}

La fréquentation et les usages de la plateforme montrent que celle-ci répond bien aujourd'hui au besoin de diagnostic territorial attendu notamment dans le cadre de la loi sur la transition énergétique. Cependant, au-delà du diagnostic, les partenaires utilisateurs et acteurs d'OPTEER mettent en avant les enjeux de plus en plus fort pour les territoires concernant la prospective.

Ces attentes sont liées notamment au besoin d'évaluer les objectifs des territoires fixés dans les schémas (SRCAE par exemple) et de mieux connaitre la trajectoire qu'ils suivent pour les atteindre. Ceci implique le développement de fonctionnalités basées sur des modèles. On est ici dans une demande de monitoring avec l'idée d'un pilotage non pas en temps réel, mais sur des pas de temps à définir qui pourraient être annuels mais avec un décalage sur la visibilité des résultats du pilotage, inhérent 
aux modèles dépendant de données statistique (populations, emplois, ...) ou de mesure (compteurs, ...).

Dans cette optique, deux projets ont d'ores et déjà été actés dans le cadre de l'évolution de l'outil OPTEER :

- A moyen terme, la co-construction, avec les acteurs des territoires, de scénarios prospectifs territorialisés (exploitable de la commune à la région) et leur intégration dans OPTEER, rendue possible suite à la refonte de l'outil en 2015, la plateforme pouvant gérer des données issues de scénarios prospectifs (mode scénario).

- A plus long terme, OPTEER ambitionne de proposer aux territoires des outils dynamiques pour la construction de scénarios.

Les réflexions menées au sein des instances de gouvernance ont par ailleurs mis en lumière un ensemble de besoins, de problématiques et d'orientations de recherche en lien avec le domaine de la fouille de données. Dans ce contexte, le support que pourrait offrir le dispositif OPTEER apparait pertinent pour mettre en ouvre un programme de travail et notamment explorer les complémentarités possibles entre fouille de données (données des compteurs communicants des opérateurs énergétiques, de téléphonie mobile par exemple) et exploitation intentionnelle de BD structurées.

Enfin, en complément de l'existence d'Infrastructures de Données Géographiques d'une part et de systèmes d'observation, producteurs d'indicateurs d'autre part, des travaux visant à développer l'interopérabilité avec des outils de management énergétique urbain ${ }^{17}$ sont également envisagés.

La convergence des points de vue exprimés dans le cadre des différentes instances de gouvernance d'OPTEER confirme l'importance de poursuivre les réflexions dans un contexte marqué par de fortes évolutions institutionnelles mais aussi avec d'énormes besoins en termes de compréhension des systèmes territoriaux (tant d'un point de vue énergétique que, plus largement, socio-environnemental, ...). Les synergies et les complémentarités entre dispositifs et organismes d'observation et d'analyse des territoires existants préalablement en Bourgogne et en FrancheComté doivent être renforcées pour une meilleure efficacité dans le cadre de la nouvelle Région. Les liens existants entre les différents acteurs, l'intégration forte à des réseaux de recherche doivent être pérennisés et valorisés pour le bénéfice d'OPTEER et des territoires l'utilisant. Enfin, l'ouverture possible en direction d'applications pratiques dans le cadre de projets et de structures publiques comme privées, notamment autour de l'évaluation et du suivi des actions mises en œuvre (indicateurs de vulnérabilité, de création de valeur partagée sur les territoires, gisements d'économies d'énergie, comptabilisation d'énergie non consommée sur les territoires (Négawatts), ...), peuvent offrir au dispositif OPTEER d'importants

\footnotetext{
${ }^{17}$ Un projet visant à développer l'interopérabilité entre l'outil OPTEER et la plateforme de management énergétique urbain MEU développée par l'EPFL est actuellement discussion.
} 
débouchés applicatifs et conforter son rôle et sa place au cœur des dispositifs informationnels existants.

\section{Conclusion}

Les systèmes énergétiques apparaissent comme de véritables instruments d'autoorganisation des territoires qu'il convient d'aborder dans une perspective de durabilité, à travers des outils performants de maîtrise de l'information par le biais d'approches de suivi, d'analyse et prospective.

OPTEER s'inscrit dans ce paradigme et son ambition est de contribuer à une meilleure compréhension des systèmes énergétiques territoriaux, malgré les difficultés que pose la conception de ce type d'outil dans un cadre contraint, tant par la pratique que par les fonctionnements institutionnels.

Fort d'un recul de sept années de mise en production de l'outil et de fonctionnement du dispositif d'observation, nous restons confiants en l'approche préconisée, plaçant les territoires au cœur de la problématique énergie/environnement/climat, persuadés que la solution réside en grande partie dans la capacité d'information, pour une meilleure connaissance des fonctionnements territoriaux et une plus grande mobilisation des acteurs de leur évolution.

Le dispositif que nous avons développé offre d'ores et déjà de nombreuses possibilités permettant d'améliorer cette connaissance, qu'il s'agisse de fonctionnalités de traitements, de représentation et de diffusion de l'information ou d'opportunité d'échanges, de mutualisation et de co-construction entre les acteurs.

La pérennisation du dispositif repose aujourd'hui sur trois grands axes de développement; la prospective, avec de forts enjeux en termes de modélisation, l'interopérabilité entre outils et notamment avec des outils de traitement de données non structurées et le renforcement de la gouvernance. Par ailleurs, les liens existant entre les partenaires scientifiques, institutionnels publics et privés doivent être confortés et valorisés pour le bénéfice de l'ensemble du dispositif OPTEER.

Dans le domaine énergétique, comment en effet vouloir infléchir des tendances si l'on ne peut concrètement mesurer l'état pressenti et au-delà les impacts des mesures engagées ? Cette connaissance ne sera acquise que si les acteurs acceptent ensemble de relever le défi énergétique en commençant par partager leurs informations, faisant ainsi sauter les verrous d'une vision par trop sectorielle. C'est sans doute à cette condition qu'une réelle vision collective des enjeux pourra émerger et que nous nous donnerons les moyens d'adapter nos modes de développement aux réalités énergétiques et environnementales de nos territoires. 


\section{Remerciements}

Les auteurs remercient la Région Franche-Comté, l'ADEME Franche-Comté et l'agglomération du Grand Dole qui ont, par leurs financements et leur accompagnement, permis d'initier ce projet, puis la Région et l'ADEME qui ont œuvré au déploiement d'OPTEER et à sa pérennisation. Un remerciement également à la DREAL Franche-Comté pour son accompagnement dans le déploiement de cette plateforme.

\section{Bibliographie}

CERTU (2011). Schéma régional du climat, de l'air et de l'énergie (SRCAE), Synthèse, Centre d'études sur les réseaux, les transports, l'urbanisme et les constructions publiques.

Chabret P. (2014). Etude comparative des dispositifs d'observations régionaux de l'énergie et des GES. Étude réalisée pour le compte de l'ADEME par I Care Environnement et Energies.

Chérix G., Capezzali M. (2013). Smart Cities ; la Métamorphose. In Urbia : les cahiers du développement urbain durable, $\mathrm{n}^{\circ} 15, \mathrm{p} .27-43$.

Couclelis H. (2010). Ontologies of geographic information, International Journal of Geographical Information Science, 24(12), p. 1785-1809.

Desjardins X. (2007). Gouverner la ville diffuse- la planification territoriale à l'épreuve, Thèse de doctorat en géographie, Université Paris I.

Fléty Y. (2014) : Vers une mise en observation des systèmes énergétiques territoriaux. Une approche géographique pour territorialiser l'énergie ». Thèse de géographie, Université de Franche-Comté.

Fléty Y., Sède-Marceau (de) M.-H. (2009). Vers une géo-ontologie pour les Systèmes Energétiques Territoriaux (SET), XVIe rencontres de Rochebrune sur les systèmes complexes naturels et artificiels : ontologie et dynamique des systèmes complexes.

Godinot S. (2011), « Les plans climat énergie territoriaux : voies d'appropriation du facteur 4 par les collectivités et les acteurs locaux ? », Développement durable et territoires [En ligne], Vol. 2, $\mathrm{n}^{\circ} 1$ |, Mars 2011.

Guarino N. (1998). Formal Ontology and Information Systems, Proceedings of the international conference on Formal Ontology in Information Systems (FOIS), Trento, Italy.

Haklay M., (2010), How good is volunteered geographical information? A comparative study of OpenStreetMap and Ordnance Survey datasets. Environment and Planning B: Planning and Design, 2010, volume 37, p. $682-703$.

IAD-Territoire Digital, (2014). Présentation technique et fonctionnelle de l'outil OPTEER. Rapport Interne.

Janin C., Grasset E., Lapostolle D., Turquin E. (2011). L'ingénierie, signe d'intelligence territoriale? Editions Economica, Collection Anthropos. 
Jebaraj S., Iniyan S. (2006). A review of energy models, Renewable and Sustainable Energy Reviews, 10(4,) p. 281-311.

Joliveau T., Noucher M., Roche S., «La cartographie 2.0, vers une approche critique d'un nouveau régime cartographique », L'Information géographique, 77/4, p. 29-46.

Kashyap V., Shah K., Sheth A. (1995), Metadata for building the Multimedia Patch Quilt. Multimedia database systems: Issues and research directions. Springer Verlag, p. $297-$ 319.

Mazaud L-A., Suaud C., Marquet S. de Sède-Marceau M-H. Noucher M., Hainaut H. Bailly B. (2017). Planification territoriale durable : opportunités offertes par les données et les outils. Numéro spécial de la Revue Internationale de Géomatique "La transition énergétique ; Enjeux informationnels et cognitifs ». 27/1, p. 11-36.

Mericskay B. (2011), «Les Sig et la cartographie à l'ère du géoweb. Vers une nouvelle génération de Sig participatifs », L'Espace géographique, 40/2, p. 142-153.

Noucher M. (2013), Infrastructures de données géographiques et flux d'information environnementale, Netcom, 27-1/2, p.120-147.

Noucher M., Gourmelon F., Laumond A., Melançon G., Pinaud B., (2016). Un cadre d'analyse des Infrastructures de Données Géographiques pour interroger la mise en réseaux des acteurs et des outils. Proceeding SAGEO : Spatial Analysis \& Geomatic, Dec 2016, Nice, France.

Palsky G. (2013), «Cartographie participative, cartographie indisciplinée », L'Information géographique, 77/4, p. 10-25.

Pornon H., (1998). Système d'Informations Géographiques, organisations géomatique et stratégies d'acteurs, Ed. l'Harmattan, Paris.

Poupeau F-M (2013). Simples territoires ou actrices de la transition énergétique ? Les villes françaises dans la gouvernance multi-niveaux de l'énergie. URBIA. Les Cahiers du développement urbain durable, (15), p. 73-90.

Roche S, C. Caron, Bédard Y., (1996), Vers une approche plus complète du rôle de la géomatique dans les organisations. Revue Internationale de Géomatique, 6/1, p. 73-92

Sède-Marceau (de) M.-H., Moine A., Thiam S. (2011). Le développement d'observatoires territoriaux, entre complexité et pragmatisme, L'Espace Géographique, 40/2, p. 117-126.

Souami T. (2007). L'intégration des technologies énergétiques dans l'action urbaine. Eclairages théoriques d'expériences européennes. Les Annales de la recherche urbaine vol. $103, \mathrm{n}^{\circ} 1$, p. 6-17.

Spaccapietra S., Parent C., Cullot N., Vangenot C. (2004). On using conceptual modeling for ontologies, Proceedings of the International Workshop on Intelligent Networked and Mobile Systems, Track 1: Ontologies for Networked Systems (ONS), WISE 2004 Workshops, Brisbane, Australia, p. 22-33.

Sui D., Elwood S, Goodchild M., (2012). Crowdsourcing Geographic Knowledge: Volunteered Geographic Information (VGI) in Theory and Practice. Springer Science \& Business Media.

Trognon L., Cayre P., Lardon S., Maury C., 2012, Ingénierie territoriale : de quoi parle-t-on ? In Trognon, Baumont, Ingrand, Lardon, Turpin, Vollet (Ed.) Productions, Gouvernance et Ingénierie Territoriales, Revue d'Auvergne, $\mathrm{N}^{\circ}$ 602-603, p.321-342. 